\title{
PROTOCOLE DE RECHERCHE SUR LES PRATIQUES DE CHASSE AUTOUR DU PARC NATUREL DE MAKIRA
}

Charlotte Spira, cspira@wcs.org

Hajaniaina Rasoloarison, hrasoloarison@wcs.org

Morgane Cournarie, mcournarie@wcs.org

Michelle Wieland, mwieland@wcs.org

David Wilkie,_dwilkie@wcs.org

\section{SOMMAIRE}

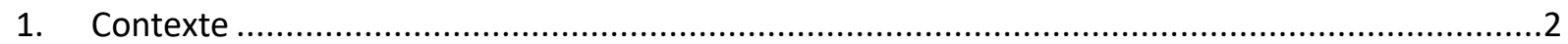

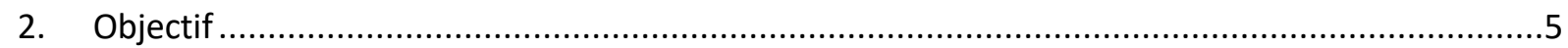

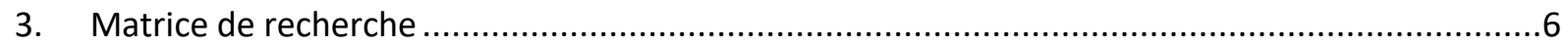

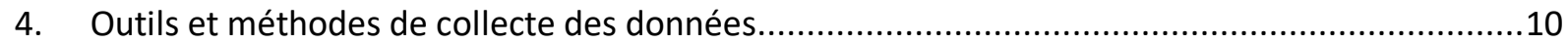

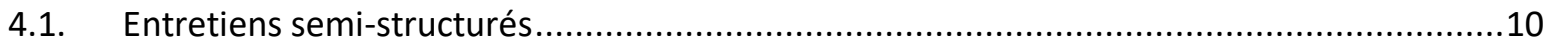

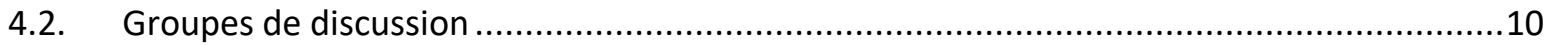

5. Méthode d'échantillonnage et sélection des participants ....................................................10

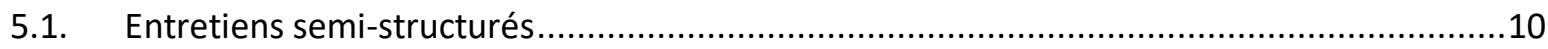

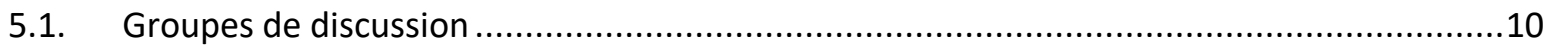

6. Consentement et protection des personnes interrogées ......................................................11

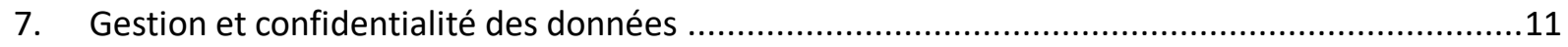

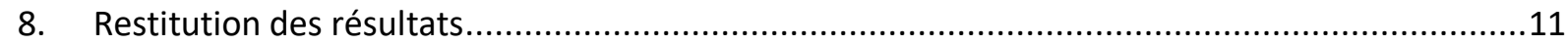

Annexe 1 - Questionnaire d'enquête individuelle sur les pratiques de chasse autour du Parc Naturel de Makira .12

Annexe 2 - Questionnaire de discussion de groupe avec les chasseurs sur les pratiques de chasse autour du Parc Naturel de Makira

Annexe 3 - Questionnaire de discussion de groupe avec les femmes de chasseurs sur leur rôle dans les activités de chasse autour du Parc Naturel de Makira 


\section{CONTEXTE}

Un facteur majeur de la chasse de viande de brousse dans le Parc Naturel de Makira et ses alentours est le manque de sources alternatives de protéines animales ${ }^{1} .23$ espèces de mammifères du parc et de sa périphérie y sont chassées pour la consommation ${ }^{1}$. Les méthodes de chasse sont spécifiquement adaptées à la capture de lémuriens (lance-pierres, lances, chiens), carnivores (lance-pierres, chiens), chauve-souris (filets), tenrecs (chiens), et potamochères (lances). La majorité des mammifères sont cependant capturés avec des pièges passifs placés sur les pistes utilisées par les animaux (pièges appelés fandrikandia), notamment les carnivores et les potamochères. De larges structures en bois avec des portes basculantes sont aussi utilisées pour la capture de potamochères. La chasse aux lémuriens fait recourt à 2 types de pièges dont le placement requiert de défricher une zone de forêt et d'y placer un tronc d'arbre ou un pont en bois sur lequel les lémuriens sont forcés de passer pour franchir la zone défrichée. Quand ces ponts connectent des fragments de forêt, les pièges sont appelés laly lava. Quand ces ponts connectent un arbre fruitier à des fragments de forêt adjacents, les pièges sont appelés laly totoko, et ciblent l'espèce qui utilise cette essence particulière d'arbre fruitier. Lors des sorties de chasse en forêt, la distance moyenne parcourue par les chasseurs est de 4,4 $\pm 2,9 \mathrm{~km}$. De nombreux agriculteurs placent des pièges juste autour de leurs maisons pour contrôler les nuisibles. En général la chasse à l'arme à feu ne semble pas être une menace majeure des communautés locales sur les populations d'animaux sauvages, mais des individus plus riches venant de villes régionales se rendent souvent dans le parc pour y chasser des lémuriens avec des armes à feu.

Pour la chasse des carnivores à Makira, les chasseurs semblent cibler des zones de forêt intactes où l'occupation, l'abondance, et la richesse spécifique sont les plus élevées².

Même si toutes les espèces de lémuriens sont protégées, la chasse d'espèces protégées est répandue ${ }^{1}$. Il est illégal de chasser le foussa Cryptoprocta ferox dans les aires protégées, mais une exception dans la loi autorise les gens à tuer tout animal qui menace leur bétail ou leurs moyens de subsistance, dont le foussa est souvent coupable en attaquant les volailles domestiques. Des évaluations quantitatives de la durabilité de la chasse de 4 espèces de lémuriens (vary noir et blanc Varecia variegata, indri Indri indri, hapalémur gris Hapalemur griseus, et lémur à front blanc Eulemur albifrons) et d'une espèce de carnivore (foussa Cryptoprocta ferox) suggèrent que la chasse de ces espèces n'est probablement pas durable ${ }^{1}$. La chasse, en particulier le piégeage, peut significativement réduire les populations animales en périphérie des zones boisées, sans autant affecter les populations totales des espèces en zones sources ${ }^{3}$. Cependant, les primates et autres espèces de grande taille et/ou à reproduction lente sont beaucoup plus susceptibles d'être chassées de manière non durable ${ }^{3}$.

La chasse pourrait être durable si la viande de brousse était la source de protéines principale d'une communauté humaine de densité de 1 personne/ $\mathrm{km}^{2}$ vivant dans la forêt, mais la densité de population de 37 personnes $/ \mathrm{km}^{2}$ autour de Makira suggère que si leur source de protéines principale

1 Golden, C.D. (2009) Bushmeat hunting and use in the Makira Forest, north-eastern Madagascar: A conservation and livelihoods issue. Oryx, 43, 386-392.

2 Farris, Z.J., Golden, C.D., Karpanty, S., Murphy, A., Stauffer, D., Ratelolahy, F., et Al. (2016) Hunting, Exotic Carnivores, and Habitat Loss : Anthropogenic Effects on a Native Carnivore Community, Madagascar The Harvard community has made this article openly available. Please share how this access benefits you . Your story matters . Citation Ac. PloS one, 120.

${ }^{3}$ FitzGibbon, C.D., Mogaka, H. \& Fanshawe, J.H. (1995) Subsistence Hunting in Arabuko-Sokoke Forest, Kenya, and Its Effects on Mammal Populations. Conservation Biology, 9, 1116-1126. 
est la viande de brousse, la chasse est probablement non durable pour de nombreuses espèces ${ }^{1,4}$. Les traits d'histoire de vie des espèces de primates et de carnivores chassées à Madagascar impliquent que même la chasse de petite échelle est une menace majeure à la conservation sur le long terme ${ }^{1}$.

98\% de la faune sauvage consommée par les ménages autour des parcs de Makira et de Masoala, situé à environ $50 \mathrm{~km}$ à l'est de Makira, est collectée directement par les chasseurs et leurs familles, et seulement $2 \%$ de la faune sauvage consommée est achetée, démontrant une quasi-absence de marché formel pour la faune sauvage dans cette zone ${ }^{5}$. Le manque de méthodes de réfrigération et de préservation efficace pousse les ménages à vendre de la viande de brousse seulement si la quantité capturée est trop importante pour être consommée dans le ménage en un jour. De ce fait, seuls les potamochères et les chauves-souris sont vendus fréquemment parce qu'ils sont respectivement trop gros pour être consommés en une journée ou capturés en très grande quantité en une nuit de chasse donnée. $91 \%$ des ménages chassent des tenrecs, $49 \%$ des lémuriens, $40 \%$ des espèces carnivores, $23 \%$ des potamochères, et $16 \%$ des chauve-souris (en particulier des roussettes). Les décisions de vendre de la viande de brousse étaient prises au jour le jour et non sur base des captures totales au cours d'une saison de chasse. A l'époque de l'étude, un ménage typique consommait en moyenne $13 \mathrm{~kg} \pm$ $0,5 \mathrm{~kg}$ de viande de brousse/an. Le prix imputé de la faune sauvage prélevée et de sa consommation étaient fortement associés, et une augmentation du prix menait à des prélèvements pour la consommation réduite. Les ménages avec de petits revenus consommaient plus de faune sauvage que de viande d'origine domestique.

Une étude menée dans un village proche du Parc National de Masoala a révélé des variations saisonnières des pratiques de chasse des mammifères forestiers, selon lesquelles les chasseurs ciblent les potamochères, les tenrecs et les lémuriens pendant l'hiver austral (mars à août), et plutôt des carnivores pendant l'été (septembre à février) ${ }^{6}$. L'effort de chasse ne variait pas significativement d'une saison à l'autre. Les variations semblent ne pas être liées à la rareté saisonnière des viandes alternatives ni aux lois et normes culturelles de chasse, mais reflètent plutôt les caractéristiques physiques et comportementales des proies, associées à une prévisibilité augmentée des déplacements des proies et davantage de chevauchement avec des zones d'habitations humaines, des champs de culture ou des animaux domestiques. La chasse s'y fait principalement pour se nourrir ou en réponse aux conflits hommes-faune résultant de la prédation des foussas sur la volaille ou de la destruction des cultures de tubercules par les potamochères, notamment. Les tenrecs et les euplérides (carnivores) sont chassés quand les femelles sont enceintes et allaitantes, et les lémuriens sont chassés après que leur progéniture soit sevrée mais avant d'être entré en période de reproduction. Seule la viande de potamochère était vendue, les animaux étant trop gros pour être entièrement consommés par le ménage avant de pourrir.

1 Golden, C.D. (2009) Bushmeat hunting and use in the Makira Forest, north-eastern Madagascar: A conservation and livelihoods issue. Oryx, 43, 386-392.

${ }^{4}$ RoBINSON, J.G. \& BENNETT, E.L. (2004) Having your wildlife and eating it too: An analysis of hunting sustainability across tropical ecosystems. Animal Conservation, 7, 397-408.

${ }^{5}$ Golden, C.D., Bonds, M.H., Brashares, J.S., Rodolph Rasolofoniaina, B.J. \& Kremen, C. (2014) Economic Valuation of Subsistence Harvest of Wildlife in Madagascar. Conservation Biology, 154, 2262-2265.

${ }^{6}$ BORGERSON, C. (2016) Optimizing conservation policy: The importance of seasonal variation in hunting and meat consumption on the Masoala Peninsula of Madagascar. Oryx, 50, 405-418. 
A Masoala, la décision de chasser des lémuriens est basée sur la pauvreté, un mauvais état de santé, et la malnutrition infantile. ${ }^{7}$ Un accès limité à des sources alternatives de protéines animales était modérément associé à la décision de chasser des lémuriens. La connaissance des lois, le niveau d'éducation, l'implication dans l'écotourisme, les valeurs culturelles traditionnelles, les préférences de goût, et les conflits hommes-faune n'intervenaient pas dans les décisions de chasser des lémuriens ${ }^{7}$. $26 \%$ des hommes du village avaient intentionnellement déployé des pièges à lémuriens et capturé des lémuriens au cours de l'année passée, dont toutes les captures étaient destinées à la consommation et aucune à la vente. Les chasseurs de lémuriens possédaient moins de bétail que les autres villageois.

A Madagascar les chauves-souris sont classées comme gibier selon la loi, et les gens les chassent habituellement dans leurs gîtes, où elles se rassemblent, ou à leurs sites d'alimentation où elles recherchent de la nourriture à proximité des habitations humaines ${ }^{8}$. Les roussettes sont particulièrement vulnérables à la capture de nuit quand elles se nourrissent de fruits et de fleurs à faible hauteur et près des villages. La nature éphémère des fruits et des fleurs implique que les chasseurs ne peuvent capturer des chauves-souris à la recherche de nourriture que pendant certaines périodes de l'années, qui sont parfois en dehors de la saison légale chasse $\left(1^{\mathrm{er}}\right.$ mai au $1^{\mathrm{er}}$ septembre pour toutes les roussettes). Dans certaines zones du pays les chasseurs relâchent les femelles enceintes ou s'abstiennent de chasser pendant la période de grossesse. Ce type de gestion est base sur la connaissance locale de l'écologie des chauves-souris et ne concorde pas avec la saison légale de chasse parce que la plupart des espèces de chauves-souris de Madagascar sont enceintes entre août et décembre.

A Masoala, les laly (zones défrichées pour la chasse aux lémuriens) sont la propriété du chasseur qui défriche la terre, et peuvent être hérités ${ }^{9}$ (Borgerson, 2015). Souvent, 2 chasseurs partagent ces zones pendant la saison de chasse, en raison de leur forte productivité et du travail nécessaire pour redéfricher des laly chaque année. Les chasseurs alternant leur utilisation des laly d'année en année, et utilisent donc rarement un laly plusieurs années de suite. Ils arrêtent d'utiliser un laly particulier quand sa productivité chute, moment auquel le laly entre dans une période d'inexploitation. Les chasseurs sélectionnent le laly qu'ils vont réétablir l'année suivante sur base du nombre d'années depuis sa dernière utilisation et du nombre de lémuriens observés dans la zone.

Malgré les recherches menées dans le passé, les connaissances sur les pratiques actuelles de chasse des communautés riveraines de Makira sont limitées. Une étude sur les pratiques de chasse est donc prévue pour dresser le profil des chasseurs, évaluer leur niveau de dépendance sur la chasse, et évaluer la durabilité des pratiques actuelles de chasse (voir détails dans la section 3. Matrice de recherche).

Ces informations permettront de caractériser les chasseurs et leurs pratiques dans les sites du programme de Gestion Durable de la Faune (Sustainable Wildlife Management, SWM), afin de bien pouvoir les cibler dans la conception et la mise en œuvre d'activités de conservation visant à réduire les pratiques de chasse non durable, et serviront de bases sur lesquelles appuyer le développement de mécanismes et plans de chasse durables avec les acteurs locaux.

\footnotetext{
7 Borgerson, C., McKean, M.A., Sutherland, M.R. \& Godfrey, L.R. (2016) Who hunts lemurs and why they hunt them. Biological Conservation, 197, 124-130. Elsevier B.V.

8 JENKINS, R.K.B. \& RACEY, P.A. (2008) Bats as bushmeat in Madagascar. Madagascar Conservation \& Development, 3.

${ }^{9}$ Borgerson, C. (2015) The Effects of Illegal Hunting and Habitat on Two Sympatric Endangered Primates. International Journal of Primatology, 36, 74-93.
} 


\section{2. ОвJECTIF}

1. Dresser le profil des chasseurs ;

2. Evaluer la place de la chasse dans les moyens de subsistance des ménages autour du parc et la dépendance des chasseurs sur cette activité ;

3. Comprendre les motivations, les difficultés, et les aspirations des chasseurs ;

4. Examiner le fonctionnement et la durabilité des pratiques de chasse.

La réalisation de ces objectifs de recherche servira de base pour le développement de mécanismes et plans de chasse durables. 


\section{Objectifs}

\section{Questions de recherche}

1. Quelles

Dresser le profil

des chasseurs

caractéristiques les

ménages de chasseurs

partagent-ils?
Hypothèses
Données

nécessaires
Méthodes d'analyse

Utilité

Type de chasseur

$(\simeq$ temps plein,

temps partiel,

Les chasseurs ont généralement un opportuniste), âge,

faible niveau d'éducation, de ethnie, religion,

relativement grands ménages, et niveau d'éducation, Modélisation par type de

sont des personnes de tout âge qui taille du ménage, chasseur

pratiquent la chasse de façon

opportuniste.

.

résident/immigré,

chasse comme

activité occupant le

plus de temps du

chasseur ou pas

\section{Evaluer la place}

de la chasse

dans les moyens

de subsistance

des ménages

autour du parc

et la

dépendance des

chasseurs sur

cette activité
2. Quelle proportion de la population dépend de la chasse pour subsiste, et dans quelle mesure

Une portion grandissante de la population chasse et dépend de la chasse pour se nourrir plus que sur toute autre activité de subsistance.
Nombre de

chasseurs, et

chasse comme

activité principale

ou pas pour se

nourrir ou pour

obtenir des

revenus

\section{Conversion du nombre de} chasseurs de chaque site en proportion de la population totale.

Nombre de groupes de discussion rapportant une augmentation du nombre de chasseurs au cours des 5 dernières années.

Pourcentage de ménages pour qui la chasse est l'activité principale qui leur permet de nourrir leur ménage ou d'obtenir des revenus.
Pouvoir précisément cibler les chasseurs et types de personnes avec lesquelles travailler pour la mise en œuvre d'activités de conservation visant à réduire la chasse non durable, et assurer que les activités soient conçues de façon à pouvoir effectivement affecter ces personnes-là.

Concevoir des activités de conservation à une échelle pertinente, qui pourront renforcer les pratiques alternatives actuelles des chasseurs et/ou les aider à réaliser leurs aspirations pour réduire leur dépendance sur la chasse. 
3. A quels problèmes et obstacles les chasseurs font-ils face dans la pratique de leurs activités de subsistance?

4. Pour quelles raisons les chasseurs chassent-ils, et à quelles autres activités de subsistance ont-ils recours quand la chasse est peu productive/rémunératr ice?

Les chasseurs font face à un certain nombre d'obstacles qui affectent la rentabilité et la durabilité de leurs activités

Comprendre les motivations, les difficultés, et les aspirations des chasseurs

\section{Quelles activités} alternatives à la chasse les chasseurs choisiraient-ils pour s'approvisionner en protéines animales et pour obtenir des revenus, s'ils le pouvaient, et quels sont les obstacles qui les en empêchent?

\section{Quels outils et} méthodes utilisent-ils
pour chasser ? Sont-ils

Examiner le fonctionnement susceptibles de

et la durabilité nécessaires

Obstacles

rencontrés par les

Liste des obstacles

chasseurs

rencontrés

\section{Raisons pour}

chasser, et activités

pratiquées par les

chasseurs pour

s'approvisionner en

protéines et pour

obtenir des

revenus pendant

ces périodes principalement pour se nourrir,

activités de subsistance pendant

les périodes où le succès des

captures diminue.

\section{La plupart des chasseurs choisirait}

l'élevage comme activité

alternative à la chasse pour

s'approvisionner en protéines et

obtenir des revenus, mais n'ont

pas accès à la terre ou aux moyens

financiers nécessaires.

\section{Activités}

alternatives que les

chasseurs

choisiraient, et

obstacles qui les en

empêchent

$\begin{array}{ll}\begin{array}{ll}\text { Les outils et techniques des } \\ \text { chasseurs traduisent généralement } \\ \text { des pratiques de chasse non }\end{array} & \begin{array}{l}\text { Matériel et } \\ \text { techniques utilisés, }\end{array} \\ \text { et fréquence et }\end{array}$

Les outils et techniques des

des pratiques de chasse non

durables, et certaines pratiques et fréquence et
Liste des activités de subsistance pratiquées pendant les périodes " basses » de chasse
Pourcentage de personnes citant l'élevage comme activité alternative souhaitée.

Pourcentage de personnes citant le manque d'accès à la terre ou le manque de moyens comme obstacles.

Identification du matériel et des techniques destructrices Connaître les pratiques actuelles de chasse et les processus décisionnels utilisés, pour discerner les 


\begin{tabular}{|c|c|c|c|c|c|}
\hline Objectifs & Questions de recherche & Hypothèses & $\begin{array}{l}\text { Données } \\
\text { nécessaires }\end{array}$ & Méthodes d'analyse & Utilité \\
\hline \multirow[t]{5}{*}{$\begin{array}{l}\text { des pratiques de } \\
\text { chasse }\end{array}$} & $\begin{array}{l}\text { menacer la viabilité } \\
\text { écologique de } \\
\text { certaines espèces? }\end{array}$ & $\begin{array}{l}\text { sont susceptibles d'être } \\
\text { particulièrement destructrices } \\
\text { pour certaines espèces/étapes de } \\
\text { vie. }\end{array}$ & $\begin{array}{l}\text { durée des sorties } \\
\text { de chasse }\end{array}$ & & \multirow{5}{*}{$\begin{array}{l}\text { pratiques qui sont durables } \\
\text { et celles qui ne le sont pas, } \\
\text { afin d'y baser les réflexions } \\
\text { et discussions au cours } \\
\text { desquelles des plans de } \\
\text { chasse durable seront } \\
\text { développés. }\end{array}$} \\
\hline & $\begin{array}{l}\text { 7. Quels risques } \\
\text { sanitaires les chasseurs } \\
\text { encourent-ils en } \\
\text { chassant? }\end{array}$ & $\begin{array}{l}\text { Une minorité de chasseurs a déjà } \\
\text { été mordue ou griffée par un } \\
\text { animal sauvage pendant la chasse, } \\
\text { les exposant potentiellement à des } \\
\text { maladies zoonotiques. }\end{array}$ & $\begin{array}{l}\text { Animaux qui ont } \\
\text { déjà mordu ou } \\
\text { griffé des } \\
\text { chasseurs, et } \\
\text { nombre de fois où } \\
\text { c'est arrivé }\end{array}$ & $\begin{array}{l}\text { Identification des espèces } \\
\text { responsables de morsures } \\
\text { ou griffures fréquentes des } \\
\text { chasseurs }\end{array}$ & \\
\hline & $\begin{array}{l}\text { 8. Quelles structures } \\
\text { locales de chasse } \\
\text { existent? }\end{array}$ & $\begin{array}{l}\text { La plupart des villages ont des } \\
\text { camps de chasse mais pas de sites } \\
\text { de vente de gibier. }\end{array}$ & $\begin{array}{l}\text { Existence et } \\
\text { emplacement } \\
\text { géographique de } \\
\text { camps de chasse et } \\
\text { sites de vente }\end{array}$ & $\begin{array}{l}\text { Cartographie et dentification } \\
\text { des villages avec } \\
\text { campements et/ou sites de } \\
\text { vente }\end{array}$ & \\
\hline & $\begin{array}{l}\text { 9. Quelles décisions } \\
\text { spatiales et } \\
\text { temporelles prennent } \\
\text { les chasseurs } \\
\text { concernant leur } \\
\text { activité de chasse, et } \\
\text { sur quoi basent-ils ces } \\
\text { décisions? }\end{array}$ & $\begin{array}{l}\text { Les décisions spatiales et } \\
\text { temporelles des chasseurs } \\
\text { reposent principalement sur des } \\
\text { facteurs environnementaux, et se } \\
\text { traduisent surtout par des } \\
\text { réactions à des déclins des } \\
\text { captures de chasse en un endroit } \\
\text { donné, reflétant une diminution } \\
\text { des populations sauvages. }\end{array}$ & $\begin{array}{l}\text { Calendrier annuel } \\
\text { de l'intensité } \\
\text { relative de la } \\
\text { chasse par espèce, } \\
\text { et aspects spatiaux } \\
\text { et temporels } \\
\text { intervenant dans } \\
\text { les décisions liées } \\
\text { aux pratiques de } \\
\text { chasse }\end{array}$ & $\begin{array}{l}\text { Identification des raisons } \\
\text { expliquant variations } \\
\text { saisonnières de chasse par } \\
\text { espèce. } \\
\text { Identification des décisions } \\
\text { basées sur l'état perçu des } \\
\text { populations sauvages. }\end{array}$ & \\
\hline & $\begin{array}{l}\text { 10. Quel est le niveau } \\
\text { de cohésion/ } \\
\text { d'organisation sociale } \\
\text { des chasseurs d'un }\end{array}$ & $\begin{array}{l}\text { Dans la plupart des sites il n'y a pas } \\
\text { d'organisation sociale des } \\
\text { chasseurs d'un même village ni de } \\
\text { villages voisins, et les chasseurs se } \\
\text { réunissent généralement }\end{array}$ & $\begin{array}{l}\text { Modalités de } \\
\text { réunions de } \\
\text { chasseurs }\end{array}$ & $\begin{array}{l}\text { Pourcentage de groupes de } \\
\text { discussions indiquant que les } \\
\text { chasseurs ne se réunissent } \\
\text { jamais. }\end{array}$ & \\
\hline
\end{tabular}




\section{Objectifs}

Questions de recherche

Hypothèses

seulement en cas de problème grave.

villages voisins?

11. Quelle est la perception des chasseurs sur l'état des populations d'animaux sauvages terrestres?

12. Quels rôles ont les femmes dans les décisions et les pratiques de chasse?
Les chasseurs perçoivent que les populations d'animaux sauvages ont chuté au cours des 5 dernières années, en particulier pour certaines espèces.

Les femmes elles-mêmes ne chassent pas mais jouent un rôle dans les décisions relatives aux espèces que leur conjoint chasse, aux ventes, et à l'utilisation des revenus des ventes.
Données

nécessaires

Méthodes d'analyse

Utilité

Liste des cas dans lesquels

les chasseurs se réunissent.

Perception des

chasseurs sur les

changements de

tailles des

populations

sauvages, par

espèce
Rôles des femmes dans les décisions et les pratiques de chasse
Nombre de groupes de discussions mentionnant une diminution, et liste des espèces concernées

Savoir dans quelle mesure et

Identification des étapes et décisions dans lesquelles les femmes ont un rôle, et de la nature de ces rôles. à quelles étapes les activités de conservation visant à réduire la chasse non durable devraient être menées avec les femmes de chasseurs. 


\section{OUTILS ET METHODES DE COLLECTE DES DONNEES}

Les questionnaires d'enquêtes ont été pilotés par le personnel de WCS dans un village proche des sites d'étude, et quelques ajustements ont été apportés à la formulation des questions.

Sous la supervision de la Responsable de la Recherche et du Suivi Ecologique et Social de WCS MaMaBaie et de l'Assistant Scientifique de Terrain SWM, les enquêtes seront menées par des enquêteurs locaux formés sur les aspects suivants :

- Concepts éthiques de la recherche sociale

- Méthodes de collecte de données sociales sur le terrain

- Sélection des participants

- Remplissage des questionnaires

- Déploiement sur le terrain

- Gestion du matériel de collecte des données

\subsection{Entretiens semi-structurés}

Un questionnaire d'enquête individuelle semi-structuré sera administré à environ 5-10\% des chasseurs de chacun des 10 sites SWM, via l'application KoboCollect sur smartphone, et visera à évaluer les paramètres qui varient parmi les chasseurs (voir questionnaire en Annexe 1 - Questionnaire d'enquête individuelle sur les pratiques de chasse autour du Parc Naturel de Makira).

Après pilotage, la durée d'une enquête individuelle est estimée à 20-30 minutes.

\subsection{Groupes de discussion}

Un questionnaire d'enquête sous format papier sera administré à des groupes de chasseurs par des enquêteurs formés (externes à WCS), et visera à évaluer les aspects de la chasse qui sont susceptibles d'être communs aux chasseurs d'un même village (voir questionnaire en 
Annexe 2 - Questionnaire de discussion de groupe avec les chasseurs sur les pratiques de chasse autour du Parc Naturel de Makira).

Un questionnaire d'enquête sous format papier sera administré à des groupes de femmes de chasseurs par des enquêtrices formées (externes à WCS), et visera à évaluer le rôle des femmes dans les activités liées à la chasse (voir questionnaire en Erreur ! Source du renvoi introuvable.).

\section{Methode D’ECHANTILlONNAGE ET SELECTION DES PARTICIPANTS}

\subsection{Entretiens semi-structurés}

Echantillonnage " snowball » stratifié par type de chasseur, où chaque personne interrogée donnera l'identité de 3 autres chasseurs à interroger : un pour qui la chasse est l'activité principale, un pour qui c'est une activité importante mais pas la principale, et un pour qui c'est une activité occasionnelle. L'échantillonnage s'arrêterait alors quand environ 5-10\% des chasseurs des sites auront été interrogés. La première personne interrogée sera identifiée grâce par les membres du Comité de Gestion ou aux tangalamena (autorité civile hautement respectée) des sites d'étude.

\subsection{Groupes de discussion}

Dans chaque COBA/ZOC, 3 groupes de discussions seront organisés :

- Un avec des vieux chasseurs ;

- Un avec des jeunes chasseurs ;

- Un avec des femmes de chasseurs.

L'équipe de recherche demandera aux membres du Comité de Gestion ou aux tangalamena de chaque site de l'orienter vers les participants visés.

\section{CONSENTEMENT ET PROTECTION DES PERSONNES INTERROGEES}

Le nom et prénom des personnes interrogées ne sera pas collecté. Les coordonnées géographiques des ménages des personnes interrogées seront collectées pour permettre de faire des analyses spatiales ultérieures.

Le consentement des autorités locales de chacun des 10 villages dans lesquelles l'étude va être menée sera recherché avant de procéder aux enquêtes. L'équipe WCS expliquera quelles sont les enquêtes qui sont prévues, quels participants sont recherchés, quel est l'objectif de l'étude, etc. Si les autorités locales acceptent, l'étude pourra procéder. Si elles refusent, la Coordinatrice du Programme SWM (Principal Investigator) se rendra sur place pour tenter de convaincre les autorités locales. En cas de maintien de refus de procéder à l'étude, WCS demandera aux autorités locales de rédiger une lettre signée et datée attestant de leur refus et de la raison de ce refus.

Le consentement de chaque personne interrogée sera recherché avant de pouvoir commencer l'enquête, à l'aide d'un texte standard en langue locale (Betsimisaraka ou Tsimihety selon le site) qui sera lu aux participants (voir version française du texte en Annexe 1 - Questionnaire d'enquête individuelle sur les pratiques de chasse autour du Parc Naturel de Makira).

Ce protocole de recherche sera soumis à l'approbation de l'Institutional Review Board de WCS. 


\section{GESTION ET CONFIDENTIALITE DES DONNEES}

Les données collectées via l'application KoboCollect sur smartphone ou tablette seront centralisées dans la base de données en ligne Kobotoolbox, protégée par un identifiant et un mot de passe, à laquelle le personnel de WCS chargé de la gestion du formulaire d'enquête et de l'analyse des données aura accès.

Les données collectées sur papier seront saisies dans un fichier Excel protégé par un mot de passe, auquel la personne chargée de l'analyse des données aura accès.

\section{RESTITUTION DES RESULTATS}

Les résultats de l'étude seront restitués auprès des participants dès leur analyse terminée, dans un format et un langage approprié. 


\section{ANNEXe 1 - QUeSTIONNAIRE D’ENQUETE INDIVIDUELLE SUR LES PRATIQUES DE CHASSE AUTOUR DU PARC}

\section{NATUREL DE MAKIRA}

\section{Présentation de l'enquête et obtention du consentement de la personne interrogée}

"Bonjour, je m'appelle Je fais partie d'un groupe d'enquêteurs qui effectuent des recherches dans ce secteur pour étudier les pratiques locales de chasse.

Votre participation à cette enquête est volontaire, a été recommandée par les autorités locales qui ont donné leur accord pour procéder à cette étude, et il n'y a ni gain ni perte associée à votre participation ou à votre refus de participer. Cette enquête devrait durer entre 20 et 30 minutes et sera tenue en privé. Si à un quelconque moment vous n'êtes plus à l'aise de répondre à mes questions, il suffira de me le signaler et nous pourrons arrêter l'enquête. Si nous interrompons l'enquête, votre fiche d'enquête sera éliminée. Pendant l'enquête, n'hésitez pas à me poser des questions ou à me signaler si une question n'est pas claire. Vous pouvez refuser de répondre à n'importe quelle question.

J'aimerais donc vous poser quelques questions par rapport aux activités et pratiques de chasse de votre ménage. Toutes les informations que vous allez me donner resteront strictement confidentielles, et ne seront utilisées que pour caractériser les pratiques des ménages du village dans lequel vous vivez, et serviront de référence pour des études ou des activités futures. Les résultats de cette étude seront utilisés pour évaluer la durabilité des pratiques de chasse dans votre village. Si les pratiques actuelles s'avèrent être non durables, WCS proposera alors aux chasseurs de votre village de les accompagner dans la conception et la mise en œuvre d'activités de conservation qui auront pour but de rendre leurs pratiques de chasse durables, afin que la faune sauvage soit une source durable de nourriture pour la communauté et que les espèces menacées d'extinction soient protégées. Les avantages de l'étude pour les personnes qui participent à l'étude seront donc d'être potentiellement appuyées dans le développement de systèmes de prélèvement durable de la faune dans leur village. Si ces systèmes sont bien appliqués, les générations futures en verront aussi les bénéfices puisqu'elles pourront continuer à reposer sur la chasse pour subsister, sans épuiser les populations d'espèces sauvages. Cela permettra également de maintenir les services écosystémiques assurés par les espèces sauvages pour les générations présentes et futures.

Les résultats de cette étude seront présentés à la communauté dans quelques mois, à une date qui sera convenue avec les autorités de la zone pour qu'un maximum d'habitants qui souhaitent connaître les résultats puissent être présents.

L'identité des participants aux enquêtes ne sera jamais communiquée à quiconque dans ou en dehors du village de quelque façon que ce soit, et nous ne collectons pas votre nom ou prénom pour protéger votre vie privée.

Est-ce que vous comprenez ce que cette enquête implique ? Si oui, accepteriez-vous de participer à cette enquête ? Soyez informé qu'il n'y a pas de bonnes ou de mauvaises réponses, seule votre opinion compte.

Accepte de participer

$\square$ Refuse de participer 
Pourrions-nous trouver un endroit confortable où je pourrais vous poser les questions de cette enquête?

Pour plus d'informations vous pouvez contacter Hajaniaina Rasoloarison au 03333181 49. »

\section{Fanazavana ilay fanadihadiana ary fahazoana ny faneken'ilay olona hohadihadiana}

« Miarahaba anao, ny anarako dia . Anisan'ireo vondron'olona mpanao fanadihadiana manao fikarohana amin'ity fizarana ity aho mba hahafantarana ny foto-piveloman'ireo tokantrano, indrindra ireo izay mivelona amin'ny ala voajanahary.

Ny fandraisanao anjara amin'izao fanadihadiana izao dia tsy an-tery, ary nahazahoana alàlana tamin'ny manampahefana eto an-toerana mba ahafahana manao izany fanadihadiana izany, ary tsy misy tombontsoa na fatiantoka manokana na handray anjara ianao na mandà ny handray anjara. Mety aharitra 20-30 minitra eo ho eo izao fanadihadiana izao izay ataontsika amin'ny toerana mitokana. Raha toa ka tsy mazoto hamaly ny fanontaniako intsony ianao eny antenantenany eny dia afaka tapahinao ny teniko ary ajanontsika ny fanadihadiana. Raha ajanontsika eny antenantenany ny fanadihadiana, dia ho foanana ny taratasy fanadihadiana niarahana taminao. Mandritra ny fanadihadiana, aza misalasala manontany ahy na milaza amiko raha misy fanontaniana tsy mazava aminao. Afaka mandà ihany koa ianao raha misy fanontaniana tsy tianao valiana.

Hanontany fanontaniana vitsivitsy aminao ary aho mikasika ny fomba fihazàna eo anivon'ny tokantranonao. Ny zavatra rehetra holazainao eto dia mijanona ho tsiambaratelo, ary tsy hampiasaina afa-tsy ho famaritana ny fombafomba ao an-tokantrano eto amin'ny vohitra fonenanao, ary amin'ny fikarohana na asa any aoriana any. Ny vokatr'izao fanadihadiana izao dia mba ahafahana mijery maharitra ny fihazàna eto amin'ny faritra misy anareo. Raha ohatra ny fihazana amin'izao fotoana izao dia vinavinaina tsy aharitra, WCS dia hanoso-kevitra anareo mpihaza eto amin'ny vohitra amin'ny fandrosoana soso-kevitra mba ampaharitra izany fihazana izany, mba ahatonga ireo biby fihaza ahazoana sakafo maharitra hoan'ny fokonolona sy mba ahavoaharo ireo biby ahiana ho lany tamingana. Ireo olona izay manaiky hanaovana fanadihadiana izany dia mety ahazo tombony amin'ny fampiroboroboana sy fampianarana ny fihazàna maharitra amin'ny vohitra misy azy. Raha tanterahina tsara ireo rafitra ireo dia handray soa ihany koa ny taranaka ho avy satria afaka manohy miantehitra amin'ny fihazana ireo biby dia tsy lany tamingana. Hanampy koa ny fitazonana ny vokatry ny haivoary homen'ny bibidia ho an'ny taranaka ankehitriny sy ho avy.

Ny vokatr'izao fanadihadiana izao dia hampahafantarina an'ity fiaraha-monina ity afaka volana vitsivitsy. Izany dia atao amin'ny fotoana izay ifanarahana amin'ny manam-pahefana eto amin'ny faritra, amin'ny fotoana hahafahan'izay rehetra liana amin'ny valin'ny fanadihadiana manatrika.

Ny mombamomba ny mpandray anjara dia tsy holazaina na amin'iza na amin'iza ao amin'ny tanàna na ivelan'ny tanàna hanaovana fanadihadiana na amin'ny fomba ahoana na amin'ny fomba ahoana, ary tsy handray ny anaranareo sy ny mombamomba anareo izahay mba hiarovana ny fiainanareo manokana. 
Azonareo ve ilay tanjona tianay ahatongava amin'ilay fanadihadiana? Raha eny, dia manaiky ve ianao fa hanaovanay ny fanadihadiana? Marihana tsara fa tsy misy valimpanontaniana ratsy fa ny fijerinareo sy ny hevitrareo no tena manan-danja.

Manaiky handray anjara

Mandà handray anjara

Afaka mahita toerana mitokana ve isika mba ahafahako mametraka fanontaniana amin'ity fanadihadiana ity?

Raha mila fanazavàna fanampiny dia afaka miantso an'i Hajaniaina Rasoloarison au 03333181 49. »

\begin{tabular}{|l|l|}
\hline Date : & \multicolumn{1}{l|}{ Coordonnées GPS : } \\
Daty : & \\
\hline Prénom et nom de l'enquêteur : & Lat : \\
\cline { 2 - 2 } $\begin{array}{l}\text { Fanampin'anarana sy anaran'ilay } \\
\text { mpanadihady : }\end{array}$ & COBA/ZOC: \\
\hline Sexe de la personne interrogée : & Village : \\
Sokajin'ilay olona hadihadiana : & Vohitra : \\
\hline Prendre une photo de la maison de la personne interrogée. \\
Alaivo sary ny tranon'ny olona hadihadiana. \\
\hline
\end{tabular}

Est-ce une vraie enquête ou une enquête d'entraînement ?

Efa fanadihadiana tena izy sa mbola fanazarana fanadihadiana?

Vraie enquête

Enquête d'entraînement

Fanadihadiana tena

Fanazarana fanadihadiana

\section{CARACTERISTIQUES GENERALES}

1. Quel âge avez-vous ?

Firy toana ianao?

2. Etes-vous né ici (village où l'enquête a lieu) ?

Teraka teto ve ianao (teto amin'ity vohitra hanaovana fanadihadiana ity) ?

Non

3. Si Q2=non, depuis quelle année êtes-vous installé ici ? Raha Q2=tsia, nanomboka oviana ianao no nonina teto ?

4. Si Q2=non, à combien de jours de marche se situe votre village d'origine ?

(si la personne interrogée ne sait pas exactement, essayer de l'estimer)

Raha Q2=tsia, maharitra dia an-tongotra hafiriana no misy ny vohitra niavianao?

(raha tsy hain'ilay olona hadihadiana mazava, ezaho vinavinaina)

5. Quelle est votre religion ?

\section{Catholique}

Inona ny fivavahanao? 


\begin{tabular}{|c|c|c|c|}
\hline & & \multicolumn{2}{|c|}{$\begin{array}{l}\square \text { Protestant (autre que FJKM ou FPVM) } \\
\square \text { Luthérien } \\
\square \text { Musulman } \\
\square \text { Animiste (religion traditionnelle) } \\
\square \text { Autre } \\
\square \text { Aucune } \\
\square \text { Katolika } \\
\square \text { Adventista } \\
\square \text { Fiangonan'i Jesoa Kristy eto Madagasikara (FJKM) } \\
\square \text { Fiangonana Protestant Vaovao Malagasy (FPVM) } \\
\square \text { Protestanta (tsy FJKM na FPVM) } \\
\square \text { Loteriana } \\
\square \text { Silamo } \\
\square \text { Fivavahana nentin-drazana } \\
\square \text { Hafa } \\
\square \text { Tsy misy }\end{array}$} \\
\hline & $\begin{array}{l}\text { Quelle est votre ethnie? } \\
\text { Inona no foko misy anao? }\end{array}$ & \multicolumn{2}{|l|}{$\begin{array}{l}\square \text { Betsimisaraka } \\
\square \text { Tsimihety } \\
\square \text { Betsileo } \\
\square \text { Sakalava } \\
\square \text { Merina } \\
\square \text { Betsimisaraka } \\
\square \text { Tsimihety } \\
\square \text { Betsileo } \\
\square \text { Sakalava } \\
\square \text { Merina }\end{array}$} \\
\hline & $\begin{array}{l}\text { Quel est votre niveau d'éducation? } \\
\text { (plus haut niveau d'éducation commencé ou termin } \\
\text { Inona ny fari-pahaizanao? } \\
\text { (mari-pahaizana ambony indridra vita na azo) }\end{array}$ & \multicolumn{2}{|l|}{$\begin{array}{l}\square \text { Aucun } \\
\square \text { Ecole primaire } \\
\square \text { Collège } \\
\square \text { Lycée } \\
\square \text { Université } \\
\square \text { Tsy nianatra } \\
\square \text { Ambaratonga I } \\
\square \text { Ambaratonga II } \\
\square \text { Ambaratonga faha III } \\
\square \text { Oniversite }\end{array}$} \\
\hline & \multicolumn{2}{|c|}{$\begin{array}{l}\text { Combien d'enfants (âgés de moins de } 18 \text { ans) vivent avec vous dans votre ménage ? } \\
\text { Firy ny zaza (latsaky ny } 18 \text { taona) monina eo anivon'ny tokantrano misy anao ? }\end{array}$} & $\cdots \cdots$ \\
\hline & \multicolumn{2}{|c|}{$\begin{array}{l}\text { Combien d'adultes (âgés de } 18 \text { ans ou plus) vivent avec vous dans votre } \\
\text { ménage (vous compris) ? } \\
\text { Firy ny olon-dehibe (18 taona na mihoatra) monina eo anivon'ny tokantrano misy } \\
\text { anao (miaraka aminao)? }\end{array}$} & $\ldots \ldots$ \\
\hline
\end{tabular}

\section{ACTIVITES DE SUBSISTANCE}

10. Est-ce que vous chassez pour ... Nourrir votre famille 


\begin{tabular}{|c|c|c|c|}
\hline \multicolumn{2}{|l|}{$\begin{array}{l}\text { (plusieurs réponses possibles) } \\
\text { Mihaza ve ianao mba ... } \\
\text { (mety misy valiny maromaro) }\end{array}$} & \multicolumn{2}{|c|}{ 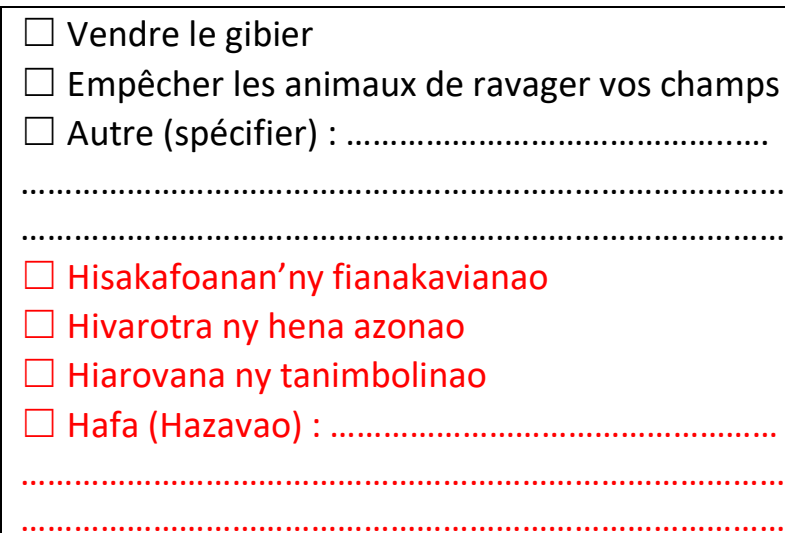 } \\
\hline \multicolumn{3}{|c|}{$\begin{array}{l}\text { 11. Parmi toutes les activités de votre ménage, est-ce que la chasse a été la source principale } \\
\text { de nourriture pour votre ménage pendant cette dernière année ? } \\
\text { Amin'ireo asa fiveloman'ny fianakavianao, ny fihazana ve no tena nahazoanareo vokatra } \\
\text { ara-tsakafo tato anatin'ny toana lasa? }\end{array}$} & $\begin{array}{l}\square \text { Oui } \\
\square \text { Non } \\
\square \text { Eny } \\
\square \text { Tsia }\end{array}$ \\
\hline \multicolumn{3}{|c|}{$\begin{array}{l}\text { 12. Si Q11=non, est-ce que vous avez besoin de chasser pour nourrir votre famille ? (c'est-à- } \\
\text { dire que sans la chasse, votre famille n'aurait pas suffisamment à manger) } \\
\text { Raha Q11=tsia, mila mihaza ve ianao mba hisakafoanan'ny fianakavianao ? (izany oe raha } \\
\text { tsy mihaza ianao, dia tsy ampy sakafo ny fianakavianao) }\end{array}$} & $\begin{array}{l}\square \text { Oui } \\
\square \text { Non } \\
\square \text { Eny } \\
\square \text { Tsia }\end{array}$ \\
\hline $\begin{array}{l}\text { 13. Quand la chasse est mauvaise, en } \\
\text { général, comment vous procurez-vous de } \\
\text { la viande ou du poisson? } \\
\text { (plusieurs réponses possibles) } \\
\text { Raha ratsy ny vokatra azo avy tamin'ny } \\
\text { fihazana, amin'ny ankapobeny, ahoana } \\
\text { no ahazoanao mihinana hena na } \\
\text { trontro? } \\
\text { (misy valiny maromaro) }\end{array}$ & \multicolumn{3}{|c|}{$\begin{array}{l}\square \text { Je n'en mange pas car je n'en ai pas besoin } \\
\square \text { Je n'en mange pas et ça me manque } \\
\square \text { J'en obtiens grâce à mon élevage } \\
\square \text { Je l'achète } \\
\square \text { Je l'échange } \\
\square \text { On m'en donne } \\
\square \text { La chasse n'est jamais mauvaise } \\
\square \text { Tsy mihinana aho satria tsy te ihinanana } \\
\square \text { Tsy mihinana aho na dia te ihinana aza } \\
\square \text { Azoko amin'ny vokatry ny fiompiana } \\
\square \text { Mividy } \\
\square \text { Manakalo } \\
\square \text { Misy manome aho } \\
\square \text { Tsara foana ny vokatry ny fihazana }\end{array}$} \\
\hline $\begin{array}{l}\text { 14. Si vous arrêtiez de chasser, quelle activité } \\
\text { choisiriez-vous de faire pour obtenir de la } \\
\text { viande ou du poisson pour vous nourrir? } \\
\text { Raha mijanona mihaza ianao, inona ny } \\
\text { asa safidianao atao mba ahazoanao hena } \\
\text { sy trondro mba hatao sakafo? }\end{array}$ & \multicolumn{3}{|c|}{$\begin{array}{l}\square \text { Pisciculture } \\
\square \text { Activité génératrice de revenus qui me permettrait } \\
\text { d'acheter des protéines animales } \\
\square \text { Je ne sais pas } \\
\square \text { Je n'ai pas envie de faire autre chose } \\
\square \text { Fiompiana } \\
\square \text { Fiompiana trondro } \\
\square \text { Asa hahazoana fidiram-bola mba hahafahana mividy } \\
\text { proteina avy amin'ny biby } \\
\square \text { Tsy haiko } \\
\square \text { Tsy te hanao zavatra hafa aho }\end{array}$} \\
\hline \multicolumn{4}{|c|}{$\begin{array}{l}\text { 15. a) Si Q14= « Je n'ai pas envie de faire autre chose ", } \\
\text { pourquoi n'avez-vous pas envie de faire autre chose? } \\
\text { Raha Q14= « Tsy te hanao zavatra hafa aho ", inona no antony } \\
\text { tsy te hanao zavatra hafa? }\end{array}$} \\
\hline
\end{tabular}




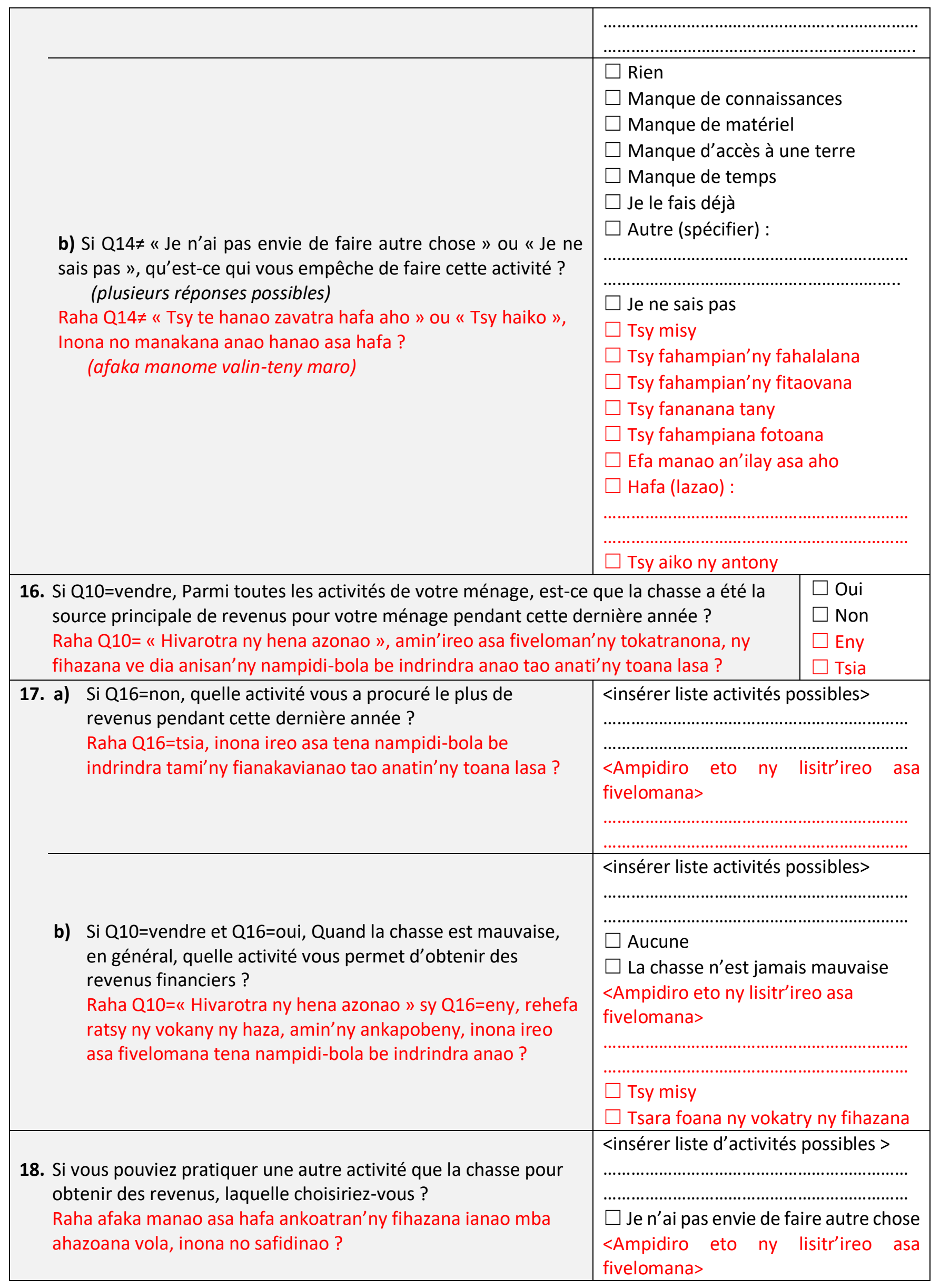


Tsy te hanao asa fivelomana hafa aho

19. a) Si Q18= "Je n'ai pas envie de faire autre chose ", pourquoi n'avez-vous pas envie de faire autre chose?

Raha Q18= "Tsy te hanao asa fivelomana hafa aho », inona no antony tsy te hanao zavatra hafa?

b) Si Q18z « Je n'ai pas envie de faire autre chose ", Qu'est-ce qui vous empêche de faire cette activité ?

(plusieurs réponses possibles)

Raha Q18* "Tsy te hanao asa fivelomana hafa aho », inona ny antony tsy hanaovanao azy?

(mety valiny maromaro)

Rien

Manque de connaissances

Manque de matériel

Manque d'accès à une terre

Manque de temps

Je le fais déjà

Autre (spécifier) :

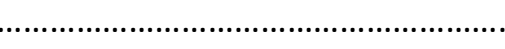

$\square$ Je ne sais pas

Tsy misy

$\square$ Tsy fahalalana

$\square$ Tsy ampy fitaovana

Tsy manana tany

Tsy misy fotoana

Efa manao an'ilay asa aho

$\square$ Hafa (hazavao) :
20. Pour vous, la chasse est ... Ho anao ve, ny fihazana dia ... $\square$ L'activité qui a occupé la plus grande partie de votre temps pendant cette dernière année.

$\square$ Une activité que vous pratiquez souvent, mais qui n'a pas occupé la plus grande partie de votre temps pendant cette dernière année.

$\square$ Une activité que vous pratiquez de temps en temps, quand l'occasion se présente (opportuniste).

$\square$ Asa fivelomana izay nandany ny fotoananao nandritra ny toana lasa.

$\square$ Asa fivelomana izay ataonao matetika, fa tsy dia nandany ny fotoananao tao anatin'ny toana lasa.

$\square$ Asa fivelomana izay fanaonao matetika, rehefa misy ny fotoana afaka hanaovana azy.

21. Si Q20ғactivité qui a occupé la plus grande partie de son temps cette dernière année, Quelle activité a occupé la plus grande partie de votre temps pendant cette dernière année ? Raha Q20 « « Asa fivelomana izay nandany ny fotoananao nandritra ny 12 volana farany ", inona ireo asa hafa izay nandany ny fotoananao tao anatin'ny toana lasa?

22. Au cours du mois dernier, combien de fois environ êtes-vous allé chasser ? Tamin'ny volana lasa, impiry ianao no nandeha nihaza? 
23. Ce nombre de sorties de chasse est-il similaire, plus faible ou plus élevé que le nombre habituel de sorties de chasse que vous faites chaque mois? lo isan'ny fotoana fihazanao io ve dia mitovy, ambaniny na mihoatra ny isan'ny fihazana mahazatra fanaonao isambolana?

24. Est-ce que quelqu'un vous a déjà demandé d'aller chasser pour lui/elle (a passé commande)?

Efa nisy olona naniraka anao hihaza ho azy ve (nanafatra haza taminao) ?

\section{$\square$ Similaire \\ $\square$ Plus faible \\ $\square$ Plus élevé \\ $\square$ Mitovy

\begin{tabular}{|c|c|}
\hline $\begin{array}{l}\text { 25. Si Q24=oui, pour quelles espèces avez-vous déjà } \\
\text { reçu des commandes? } \\
\text { Raha Q24=eny, karazana biby inona avy no efa } \\
\text { nisy nanafatra taminao? }\end{array}$ & $\begin{array}{l}<\text { insérer liste espèces avec noms locaux et images> } \\
\square \text { Pas d'espèce particulière commandée }\end{array}$ \\
\hline & (1) \\
\hline $\begin{array}{l}\text { 26. Est-ce que des animaux sauvages ont déjà ravagé } \\
\text { vos cultures? } \\
\text { Efa nisy biby dia ve nanimba ny volinao? }\end{array}$ & $\begin{array}{l}\square \text { Oui } \\
\square \text { Non } \\
\square \text { Je n'ai pas de champ } \\
\square \text { Eny } \\
\square \text { Tsia } \\
\square \text { Tsy manana tanimboly aho }\end{array}$ \\
\hline $\begin{array}{l}\text { 27. Si Q26=oui, quelles sont les espèces d'animaux } \\
\text { sauvages qui ont déjà ravagé vos cultures? } \\
\text { Raha Q26=eny, inona avy ireo biby dia nanimba } \\
\text { ny volinao? }\end{array}$ & $\begin{array}{l}<\text { insérer liste d'espèces avec noms locaux et photos > } \\
<\text { ampidiro eto ny lisitr'ireo biby sy ny anarany amin'ny } \\
\text { fitenim-paritra }\end{array}$ \\
\hline $\begin{array}{l}\text { 28. Si Q26=oui, que faites-vous pour empêcher ces } \\
\text { animaux de ravager vos cultures? } \\
\text { Raha Q26=eny, inona no ataonao mba } \\
\text { hanakanana ireo biby ireo tsy handrava ny } \\
\text { volinao? }\end{array}$ & $\begin{array}{l}\square \text { Rien } \\
\square \text { Creuse un fossé autour des champs } \\
\square \text { Clôture en bois/bambou autour des champs } \\
\square \text { Pose de pièges autour des champs } \\
\square \text { Chasse préventive en forêt } \\
\square \text { Surveillance visuelle des champs } \\
\square \text { Chiens pour surveiller les champs } \\
\square \text { Epouvantail } \\
\square \text { Autre (spécifier) : ............................ } \\
\square \text { Tsy misy }\end{array}$ \\
\hline
\end{tabular}




\begin{tabular}{|l|l|l|}
\hline & $\square$ Mangady lavaka manodidina ny tanimboly \\
& $\square$ Fefy hazo/bambo \\
& $\square$ Fametrahana fandrika manodidina ny tanimboly \\
& $\square$ Fihazana mialoha any an'ala \\
& $\square$ Fiambenana ny tanimboly \\
& $\square$ Alika mphiandry tanimboly \\
& $\square$ Sary fampitahorana biby/mpiambim-pody \\
& $\square$ Hafa (lazao) : .................. \\
\hline 29. Quand les gens placent des pièges autour de leur champ pour empêcher les animaux de & $\square$ Oui \\
détruire leurs cultures, est-ce que vous considérez cette pratique comme de la chasse ? & $\square$ Non \\
Rehefa mametraka fandrika manodidina ny sahany mba hiarovana ny fanimban'ny biby ny & $\square$ Eny \\
tanim-boly, sokajianareo ho fihazàna ve izany fanao izany ? & $\square$ Tsia \\
\hline
\end{tabular}

\section{TECHNIQUES DE CHASSE}

30. Quelles sont les techniques que vous utilisez pour la chasse?

Inona avy ny paik'ady ataonao amin'ny fihazana?

$\square$ Pose de piège autour des champs
$\square$ Pose de piège autour de la maison
$\square$ Pièges dans la forêt
$\square$ Laly lava
$\square$ Laly totoko
$\square$ Lance
$\square$ Lance-pierres
$\square$ Filet
$\square$ Arme à feu
$\square$ Chasse avec chiens
$\square$ Autre (préciser) : .................................................
$\square$ Fametrahana fandrika manodidina ny tanimboly
$\square$ Fametrahana fandrika manodidina ny trano
fonenana
$\square$ Fandrika anaty ala
$\square$ Laly lava
$\square$ Laly totoko
$\square$ Saboha
$\square$ Antsamotady
$\square$ Fandrika harato
$\square$ Basy
$\square$ Mihaza miaraka amin'ny amboa
$\square$ Hafa (lazao) : ...................................................

31. Quand vous chassez, en général, est-ce que vous ciblez une ou des espèces particulières, ou est-ce que vous ciblez tous les animaux de la forêt?

Rehefa mihaza ianareo, amin'ny ankapobeny, misy biby fihaza ve kendrenao manokana sa izay biby rehetra fihaza any anaty ala?

$\square$ Chasse ciblée

$\square$ Chasse sans discernement

Misy sokajiny

$\square$ Tsy misy sokajiny

\section{Si Q31=chasse ciblée, quelle(s) $\quad$ <insérer liste espèces terrestres>} espèce(s) ciblez-vous ?

Raha Q31=Misy sokajiny, manokana, inona ireo biby ireo? 


\begin{tabular}{|c|c|c|c|c|c|c|}
\hline & \multicolumn{6}{|c|}{ <ampidiro eto ny lisitr'ireo biby> } \\
\hline \multicolumn{2}{|l|}{$\begin{array}{l}\text { 33. Quelles espèces avez-vous chassé l'année } \\
\text { passée ? } \\
\text { Inona daholo ny biby nohazainareo tamin'ny } \\
\text { taona lasa? }\end{array}$} & \multicolumn{5}{|c|}{ <insérer liste espèces terrestres> } \\
\hline \multicolumn{2}{|c|}{$\begin{array}{l}\text { 34. Avez-vous déjà été mordu ou griffé par un animal } \\
\text { sauvage pendant la chasse? } \\
\text { Efa nisy fotoana ve ianao voakaikitra na } \\
\text { voarangotran'ny biby dia nandritra ny fihazana? }\end{array}$} & \multicolumn{5}{|l|}{$\begin{array}{l}\square \text { Oui } \\
\square \text { Non } \\
\square \text { Eny } \\
\square \text { Tsia }\end{array}$} \\
\hline \multicolumn{2}{|c|}{$\begin{array}{l}\text { 35. Si Q34=oui, par quel animal sauvage, le plus } \\
\text { souvent? } \\
\text { Raha Q34=eny, biby dia inona matetika? }\end{array}$} & \multicolumn{5}{|c|}{ (n) } \\
\hline \multicolumn{7}{|c|}{$\begin{array}{l}\text { 36. Si Q34=oui, combien de fois avez-vous été mordu ou griffé par un animal sauvage pendant cette } \\
\text { dernière année ? } \\
\text { Raha Q34=eny, impiry ianao no voakaikitra na voarangotran'ny biby dia tao anatin'ny toana lasa? }\end{array}$} \\
\hline \multicolumn{3}{|c|}{$\begin{array}{l}\text { 37. Préférez-vous chasser la journée ou la nuit ? } \\
\text { Tianao kokoa ve ny mihaza amin'ny andro sa amin'ny alina? }\end{array}$} & $\begin{array}{l}\square \text { Journe } \\
\square \text { Nuit } \\
\square \text { Pas de } \\
\square \text { Andro } \\
\square \text { Alina } \\
\square \text { Mety }\end{array}$ & préférenc & & \\
\hline \multicolumn{7}{|l|}{$\begin{array}{l}\text { 38. Pourquoi ? } \\
\text { Nahoana? }\end{array}$} \\
\hline \multicolumn{7}{|c|}{ (1) } \\
\hline \multicolumn{4}{|c|}{$\begin{array}{l}\text { 39. Combien de jours/nuits d'affilée au minimum êtes-vous déjà resté en forêt pour } \\
\text { chasser? } \\
\text { Firy andro/alina mitohy farafahakeliny ianao no efa nijanona anatiala nihaza? }\end{array}$} & \multicolumn{3}{|c|}{$\begin{array}{l}\text {........ nuits/jours } \\
\text {.......... andro/alina }\end{array}$} \\
\hline \multicolumn{4}{|c|}{$\begin{array}{l}\text { 40. Combien de jours/nuits d'affilée au maximum êtes-vous déjà resté en forêt pour } \\
\text { chasser ? (ce nombre peut être égal au nombre minimum de jours/nuits passés } \\
\text { en forêt, si la personne reste toujours la même durée) } \\
\text { Firy andro/alina mitohy farafahabetsany ianao no efa nijanona anatiala nihaza? } \\
\text { (mety mitovy amin'ny andro farafahakeliny ve no efa nijanona tany an'ala } \\
\text { amin'ny andro/alina, raha ohatra ilay olana mijanona amin'ny fotoana mitovy) }\end{array}$} & .............. nu ar & & \\
\hline \multicolumn{5}{|c|}{$\begin{array}{l}\text { 41. Est-ce qu'il y a des moments/périodes particulières de l'année pendant lesquelles votre } \\
\text { activité de chasse s'intensifie (passe plus de temps / va plus souvent/ pose plus de pièges) ? } \\
\text { Misy fotoana/vaninandro manokana mandritra ny taona ve ianao mihaza bebe kokoa } \\
\text { (mandany fotoana bebe kokoa/mandeha matetika/mametraka fandrika bebe kokoa)? }\end{array}$} & $\begin{array}{l}\square \mathrm{O} \\
\square \mathrm{N} \\
\square \mathrm{Er} \\
\square \mathrm{Ts}\end{array}$ & \\
\hline $\begin{array}{l}\text { 42. Si Q41=oui, lesquelles ? } \\
\text { Raha Q41=eny, amin'ny fotoana inona? }\end{array}$ & $\begin{array}{l}\square \text { Marią } \\
\square \text { Naissa } \\
\square \text { Enterr } \\
\square \text { Retour } \\
\square \text { Fêtes r }\end{array}$ & $\begin{array}{l}\text { se } \\
\text { nce } \\
\text { ement } \\
\text { nement c } \\
\text { lationales }\end{array}$ & es (No & âques, & & etc.) \\
\hline
\end{tabular}




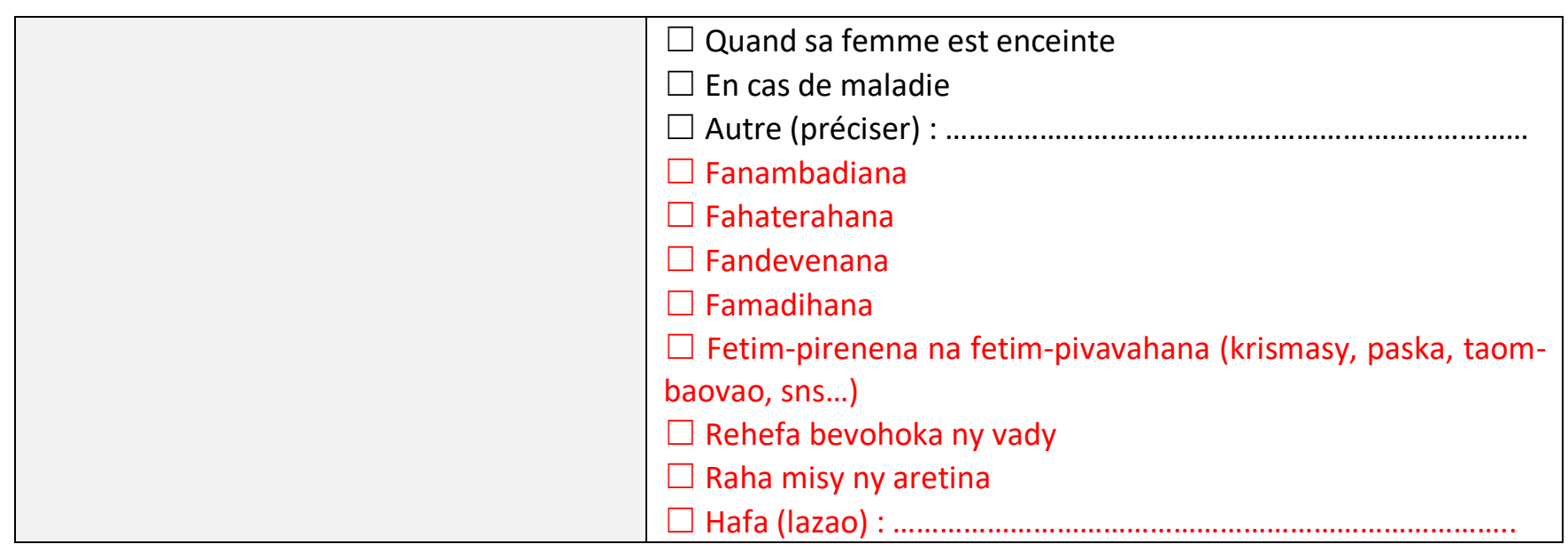




\section{Présentation du groupe de discussion et obtention du consentement des participants}

"Bonjour, nous sommes et

Nous effectuons des recherches dans ce secteur pour étudier les pratiques locales de chasse.

Votre participation à cette discussion est volontaire, a été recommandée par les autorités locales qui ont donné leur accord pour procéder à cette étude, et il n'y a ni gain ni perte associée à votre participation ou à votre refus de participer. Cette discussion devrait durer environ $X$ minutes/heures. Si à un quelconque moment vous n'êtes plus à l'aise de répondre à nos questions, il suffira de nous le signaler et vous pourrez quitter la discussion. Pendant les discussions, n'hésitez pas à nous poser des questions ou à nous signaler si une question n'est pas claire.

Nous aimerions donc vous poser quelques questions par rapport aux pratiques locales de chasse. Toutes les informations que vous allez nous donner resteront strictement confidentielles, et ne seront utilisées que pour caractériser les pratiques du village dans lequel vous vivez, et serviront de référence pour des études ou des activités futures. Les résultats de cette étude seront utilisés pour évaluer la durabilité des pratiques de chasse dans votre village. Si les pratiques actuelles s'avèrent être non durables, WCS proposera alors aux chasseurs de votre village de les accompagner dans la conception et la mise en œuvre d'activités de conservation qui auront pour but de rendre leurs pratiques de chasse durables, afin que la faune sauvage soit une source durable de nourriture pour la communauté et que les espèces menacées d'extinction soient protégées. Les avantages de l'étude pour les personnes qui participent à l'étude seront donc d'être potentiellement appuyées dans le développement de systèmes de prélèvement durable de la faune dans leur village. Si ces systèmes sont bien appliqués, les générations futures en verront aussi les bénéfices puisqu'elles pourront continuer à reposer sur la chasse pour subsister, sans épuiser les populations d'espèces sauvages. Cela permettra également de maintenir les services écosystémiques assurés par les espèces sauvages pour les générations présentes et futures.

Les résultats de cette étude seront présentés à la communauté dans quelques mois, à une date qui sera convenue avec les autorités de la zone pour qu'un maximum d'habitants qui souhaitent connaître les résultats puissent être présents.

L'identité des participants aux groupes de discussions ne sera jamais communiquée à quiconque dans ou en dehors du village de quelque façon que ce soit, et nous ne collectons pas votre nom ou prénom pour protéger votre vie privée.

Est-ce que vous comprenez ce que cette enquête implique ? Si oui, accepteriez-vous de participer à cette discussion ? Soyez informé qu'il n'y a pas de bonnes ou de mauvaises réponses, seule votre opinion compte.

Pour plus d'informations vous pouvez contacter Hajaniaina Rasoloarison au 03333181 49. » 


\section{Fanazavana ilay fanadihadiana ary fahazoana ny faneken'ilay olona hohadihadiana}

«Miarahaba anao, ny anarako dia Anisan'ireo vondron'olona mpanao fanadihadiana manao fikarohana amin'ity fizarana ity aho mba hahafantarana ny foto-piveloman'ireo tokantrano, indrindra ireo izay mivelona amin'ny ala voajanahary. Ny fandraisanao anjara amin'izao fanadihadiana izao dia tsy an-tery, ary nahazahoana alàlana tamin'ny manampahefana eto an-toerana mba ahafahana manao izany fanadihadiana izany, ary tsy misy tombontsoa na fatiantoka manokana na handray anjara ianao na mandà ny handray anjara. Mety aharitra X minitra eo ho eo izao fanadihadiana izao izay ataontsika amin'ny toerana mitokana. Raha toa ka tsy mazoto handray anjara amin'ny fahadihadiana ianao dia afaka miala amin'ny adihevitra izay atao. Mandritra ny fanadihadiana, aza misalasala manontany ahy na milaza amiko raha misy fanontaniana tsy mazava aminao. Afaka mandà ihany koa ianao raha misy fanontaniana tsy tianao valiana.

Hanontany fanontaniana vitsivitsy aminao ary aho mikasika ny fomba fihazàna eo anivon'ny tokantranonao. Ny zavatra rehetra holazainao eto dia mijanona ho tsiambaratelo, ary tsy hampiasaina afa-tsy ho famaritana ny fombafomba ao an-tokantrano eto amin'ny vohitra fonenanao, ary amin'ny fikarohana na asa any aoriana any. Ny vokatr'izao fanadihadiana izao dia mba ahafahana mijery maharitra ny fihazàna eto amin'ny faritra misy anareo. Raha ohatra ny fihazana amin'izao fotoana izao dia vinavinaina tsy aharitra, WCS dia hanoso-kevitra anareo mpihaza eto amin'ny vohitra amin'ny fandrosoana soso-kevitra mba ampaharitra izany fihazana izany, mba ahatonga ireo biby fihaza ahazoana sakafo maharitra hoan'ny fokonolona sy mba ahavoaharo ireo biby ahiana ho lany tamingana. Ireo olona izay manaiky hanaovana fanadihadiana izany dia mety ahazo tombony amin'ny fampiroboroboana sy fampianarana ny fihazàna maharitra amin'ny vohitra misy azy. Raha tanterahina tsara ireo rafitra ireo dia handray soa ihany koa ny taranaka ho avy satria afaka manohy miantehitra amin'ny fihazana ireo biby dia tsy lany tamingana. Hanampy koa ny fitazonana ny vokatry ny haivoary homen'ny bibidia ho an'ny taranaka ankehitriny sy ho avy.

Ny vokatr'izao fanadihadiana izao dia hampahafantarina an'ity fiaraha-monina ity afaka volana vitsivitsy. Izany dia atao amin'ny fotoana izay ifanarahana amin'ny manam-pahefana eto amin'ny faritra, amin'ny fotoana hahafahan'izay rehetra liana amin'ny valin'ny fanadihadiana manatrika.

Ny mombamomba ny mpandray anjara amin'ny fanadihadiana iarahana dia tsy holazaina na amin'iza na amin'iza ao amin'ny tanàna na ivelan'ny tanàna hanaovana fanadihadiana na amin'ny fomba ahoana na amin'ny fomba ahoana, ary tsy handray ny anaranareo sy ny mombamomba anareo izahay mba hiarovana ny fiainanareo manokana.

Azonareo ve ilay tanjona tianay ahatongava amin'ilay fanadihadiana ? Raha eny, dia manaiky ve ianao fa hiady hevitra miaraka isika ? Marihana tsara fa tsy misy valimpanontaniana ratsy fa ny fijerinareo sy ny hevitrareo no tena manan-danja.

Raha mila fanazavàna fanampiny dia afaka miantso an'i Hajaniaina Rasoloarison au 03333181 49. »

\begin{tabular}{l|l}
\hline Date : & $\begin{array}{l}\text { Nom de la COBA/ZOC : } \\
\text { Anaran'ny VOI/ZOC : }\end{array}$ \\
\hline $\begin{array}{l}\text { Paty : } \\
\text { discussion : }\end{array}$ & Village/hameau : \\
Tanàna/Zana-tanàna :
\end{tabular}


Anarana sy fanampin'anaran'ny

mpanelanelana :

Prénom et nom de la personne qui remplit le formulaire :

Anaran'ny olona mpandray an-tsoratra :

Heure de début :

Ora nanombohana :
Nombre et sexe des personnes dans le groupe de discussion (ne pas compter les facilitateurs de la discussion) :

Isa sy sokajin'ny olona ao anatin'ny fanadihadiana (tsy hisaina ny mpanentana ny ady hevitra) :

hommes

femmes

Lahy ; .Vavy

Heure de fin :

Ora namaranana :

\section{ASPECTS GENERAUX : CHANGEMENTS OBSERVES ET TECHNIQUES DE CHASSE}

1. Dans votre village/hameau, selon vous, combien y a-t-il de personnes pour qui la chasse occupe la majeure partie de leur temps?

Eto amin'ny faritra misy anareo, firy eo ho eo, ny isan'ny olona mpihaza amin'ny ampahan'ny fotoanany?

2. Selon vous, est-ce que qu'il y a moins, autant, ou plus de chasseurs qui dépendent de la chasse pour se nourrir, que quand vous avez commencé à chasser ? (une seule réponse possible)

Araka ny fahitanareo azy, firy eo ho eo, ny mpihaza izay hitanareo nanao izany asa izany mba hivelomana, rehefa nanomboka ny fihazana ianareo ? (valiny iray ihany no marina)

$\square$ Pas de changement

Moins de chasseurs

$\square$ Plus de chasseurs

$\square$ Ne sait pas

$\square$ Tsy misy fiovana

$\square$ Tsy dia betsaka loatra

$\square$ Maro mpihaza

Tsy manan-kevitra

Plus d'animaux

Moins d'animaux

3. Au cours des 5 dernières années, avez-vous observé des changements au niveau des populations d'animaux sauvages ?

Expliquer.

Tao anatin'ny dimy taona farany, nisy fiovana ve ny biby dia any anaty ala ?

Hazavao. 
4. Au cours des 5 dernières années, avez-vous observé des changements au niveau de la prédation des cultures?

Si oui, expliquer.

Tao anatin'ny dimy taona farany, nahavoamarika fiovana teo amin'ny biby mihinana/manimba ny tanimboly nareo ve ianareo ?

Raha eny, hazavao.
$\square$ Oui
$\square$ Non
$\square$ Ne savent pas
$\square$ Eny
Tsia
$\square$ Tsy fantatro

5. Qu'est-ce qu'il faudrait faire, selon vous, pour empêcher les animaux de détruire les cultures et les élevages, sans avoir à utiliser des pièges?

Araka ny hevitrareo, inona no tokony atao mba hisakanana ireo biby mpanimba voly na mpihaza ny biby fiompy raha tsy mampiasa fandrika?

6. Quels problèmes/obstacles rencontrez-vous dans votre activité de chasse ?

(matériel en mauvais état, mauvais rendement, compétition avec les autres chasseurs du village, compétition avec les chasseurs d'autres villages, autre (préciser), aucun)

Inona ny olana/sakana amin'ny asa fihazana ataonareo ?

(ratsy ny fitaovana fihazana, tsy misy vokatra azo, fifaninanana amin'ireo mpihaza avy amin'ny mpiray tanàna, fifaninanana avy amin'ireo mpihaza avy amin'ny tanàna hafa, hafa (mariho), tsy misy) 


\begin{tabular}{|l|}
\hline \\
\hline \\
\hline
\end{tabular}

\section{ASPECTS SPATIAUX ET TEMPORELS DE LA CHASSE}

7. Pouvez-vous décrire les variations dans l'espace et dans le temps des activités de chasse, au cours d'une même année et au fil de plusieurs années ?

Comment est-ce que vous choisissez les endroits où vous allez chasser?

Afaka hazavainareo ve, ny vanimpotoana sy ny toerana fanaovanareo ny fihazàna, mandritra ny taona sy mandritra ny taona maromaro?

Ahoana no isafidiananareo ireo toerana fihazàna? 


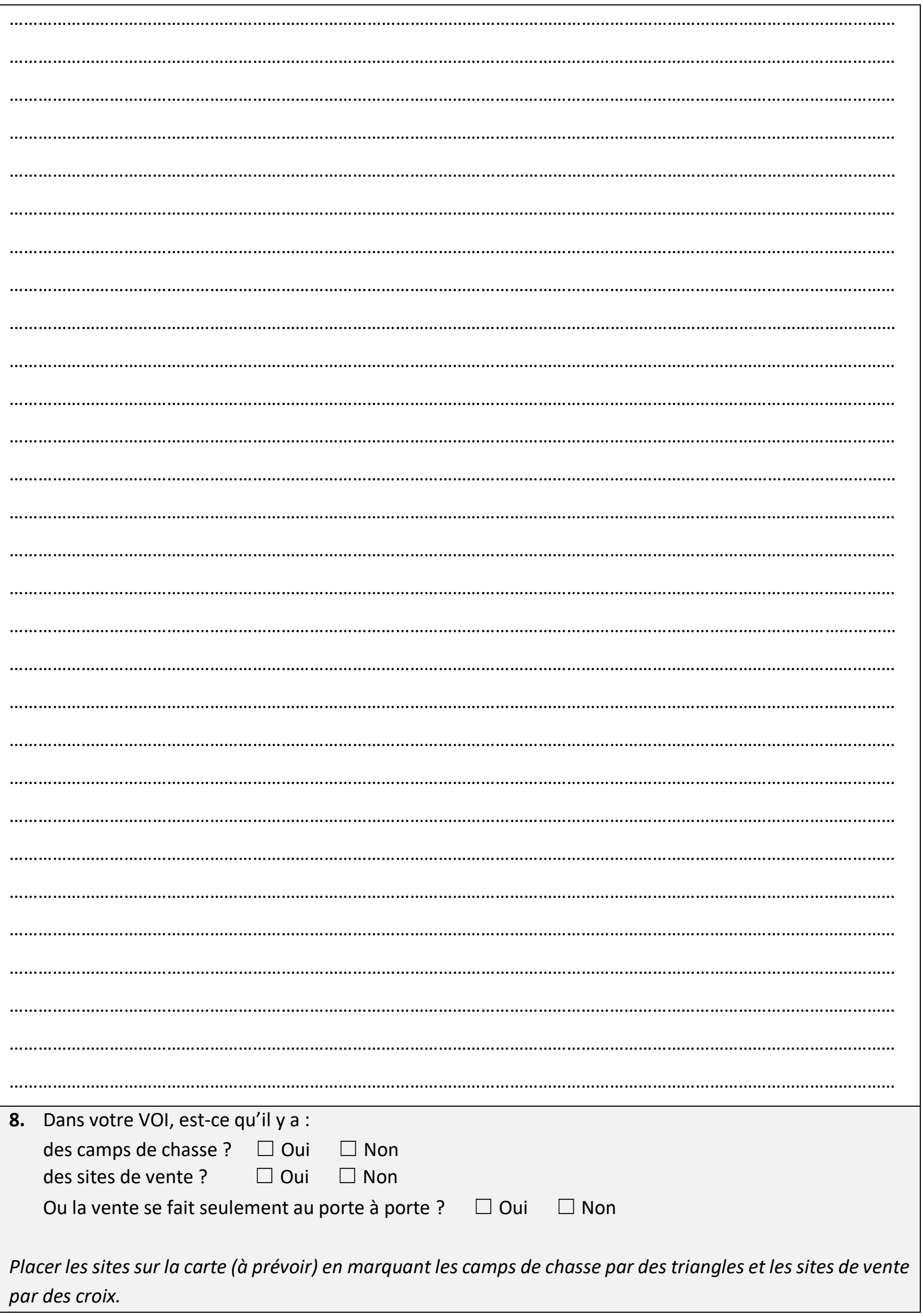


Ao amin'ny VOI misy anareo, misy toerana misy

Toerana manokana fihazan? $\square$ Eny $\quad \square$ Tsia

Toerana manokana fivarotana vokatry ny fihazàna ? $\square$ Eny $\quad \square$ Tsia

Sa ireo fihazàna ve dia atao isan-trano ? $\square$ Eny $\quad \square$ Tsia

Ataovy amin'ny sarin-tany (efa nomanina) ary mariho ireo toerana manokàna fihazàna amin'ny telozoro sy ireo toerana fivarotana amin'ny $x$.

9. Est-ce qu'il y a des endroits particuliers où vous ne voulez jamais aller chasser ? Misy toerana manokana ve izay tsy tianareo mihitsy ny mihaza any amin'izany toerana izany?

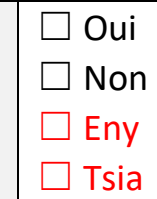

10. Si Q9=oui, les pointer sur la carte (à prévoir) et demander pourquoi. (Fady, interdit par la loi/dina, pas d'animaux là-bas, parcelle de quelqu'un d'autre, etc.)

Numéroter les endroits pointés sur la carte et écrire la raison ci-dessous.

Raha Q9=Eny, mariho amin'ny sarintany (efa nomanina) ary hanontanio oe satria nahoana.

(Fady, raràn'ny lalàna/dina, tsy misy biby amin'ireo toerana ireo, na amin'ireo tany ireo, tanin'olona, sns.)

Alaharo ireo toerana izay nomarihina tamin'ny sarintany ary soraty eo ambany ny antony.

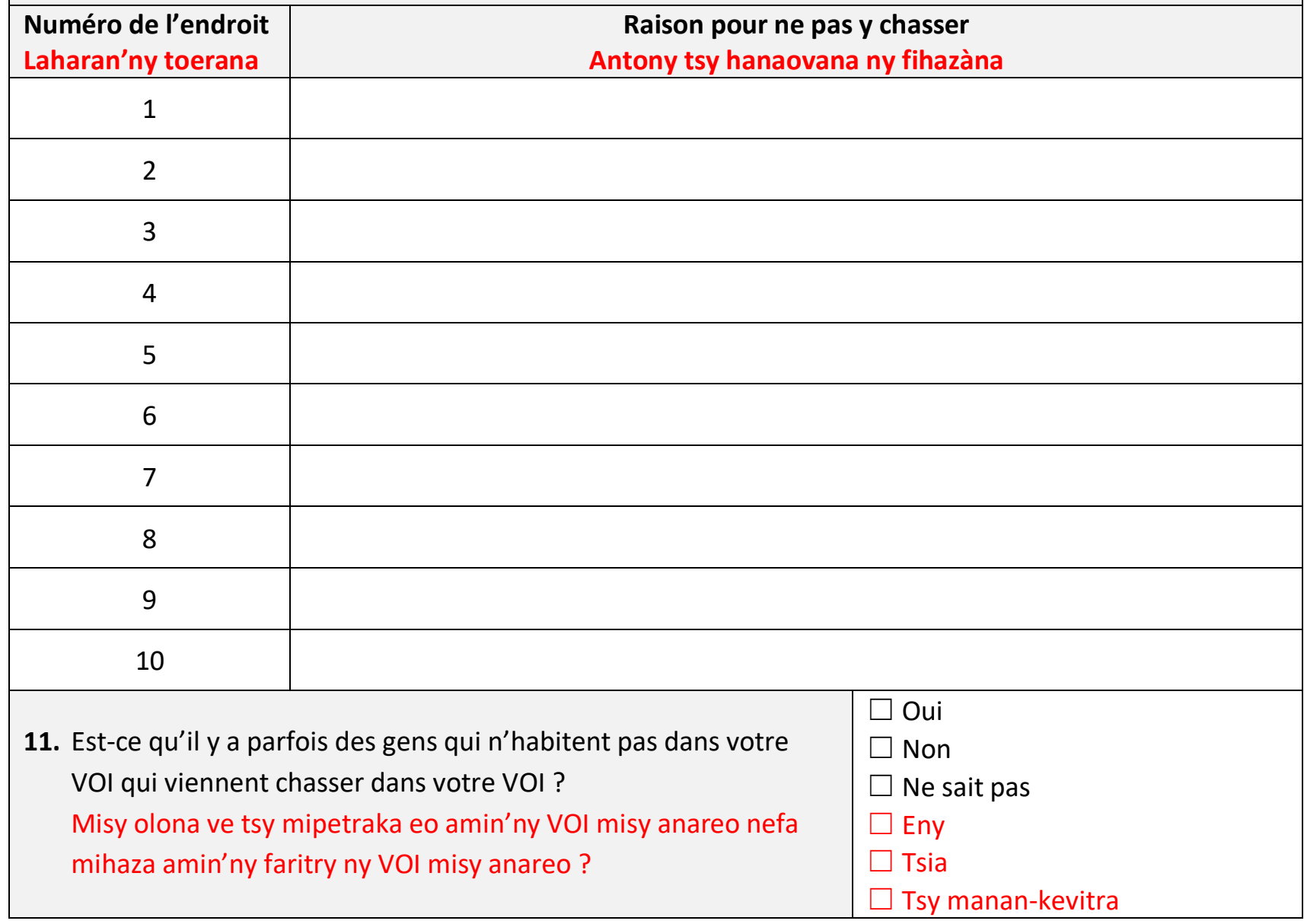


12. Si Q11=oui, est-ce qu'ils viennent de VOI voisins ou de la ville?

(plusieurs réponses possibles)

Raha QErreur ! Source du renvoi

introuvable.1=eny, avy amin'ireo VOI

manodidina ve ny fiaviany sa avy any antanàn-dehibe ?

(mety misy valiny maromaro)
VOI voisins

Ville (préciser laquelle) :

$\square$ Autre (préciser) :

Ne sait pas

VOI manodidina

Tanàn-dehibe (mariho) :

Hafa (mariho) :

$\square$ Tsy fantatro

13. Si QErreur! Source du renvoi introuvable.1=oui, selon vous, est-ce que c'est une bonne chose, une mauvaise chose, ou ça n'a pas d'importance?

Expliquer et préciser pourquoi.

Raha Q11=eny, araka ny fiheveranareo azy, zavatra tsar ave izany, sa zavatra ratsy, za tsy dia misy lanjany?

Hazavao sy hamarino ny antony. 
14. Parmi les espèces de lémuriens suivantes, en général, lesquelles sont le plus chassées ?

a) Demander aux participants de placer des haricots sur les photos, en mettant plus de haricots sur les photos des espèces les plus chassées, et moins de haricots sur les espèces les moins chassées.

b) Ecrire dans les cases le nombre de haricots qui ont été placés sur la photo de l'espèce.

Amin'ireto varika manaraka ireto, amin'ny ankapobeny, iza no tena hazainareo indrindra ?

a) Angataho ny mpandray anjara mba hanisy tsaramaso amin'ireo sary, ary asiana tsaramaso betsaka amin'reo varika tena be mpihaza sy tsaramaso vitsivitsy amin'ireo vitsy mpihaza.

b) Soratana eo amin'ny efa-joro ny isan'ny tsaramaso napetraky ny mpandray anjara tamin'ny sarin'ilay biby.

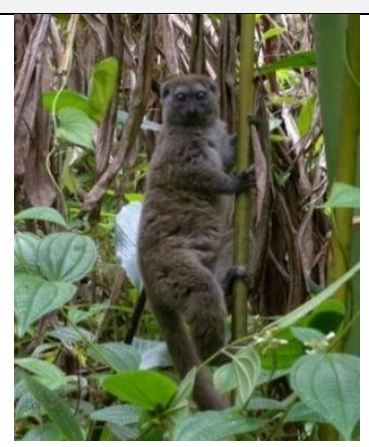

Bokombolo Hapalemur griseus

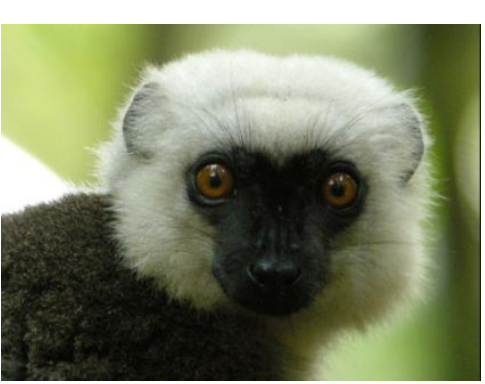

Varikosy fotsi loha Eulemur albifrons

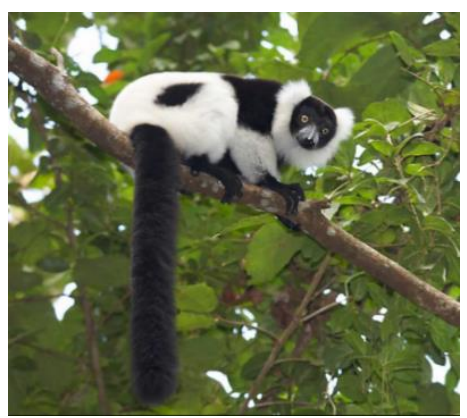

Varikandana Varecia variegata

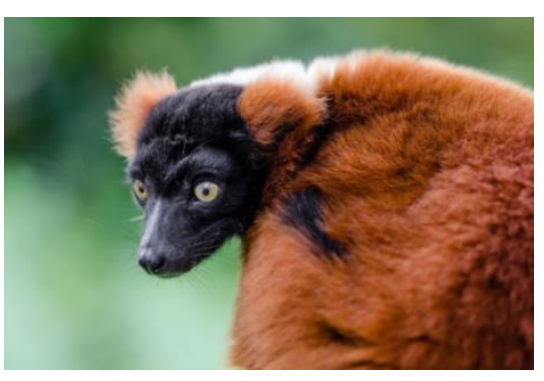

Varignena Varecia rubra 


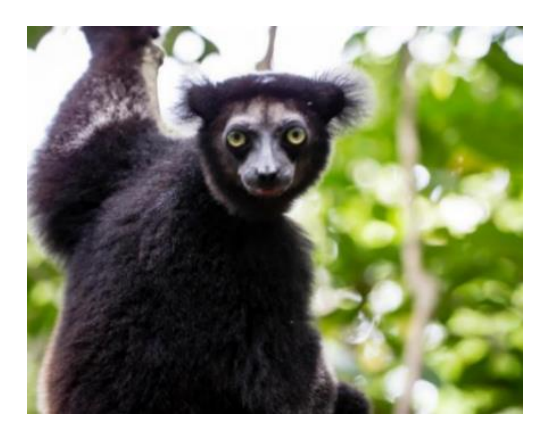

Babakoto Indri indri

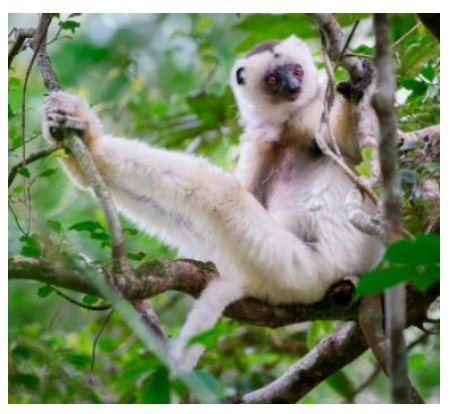

Simpona Propithecus candidus

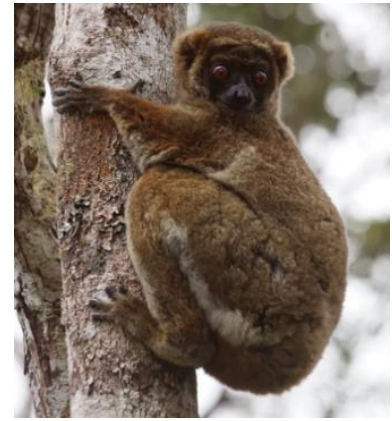

Ampongy, Fotsife Avahi laniger

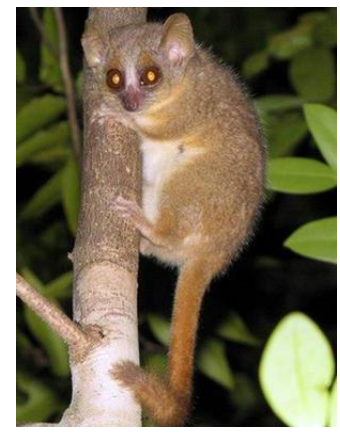

Tsidy, Kandrandra Microcebus sp.

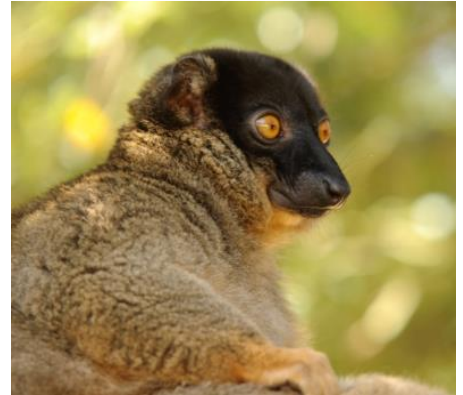

Varikosy mainty loha Eulemurfulvus

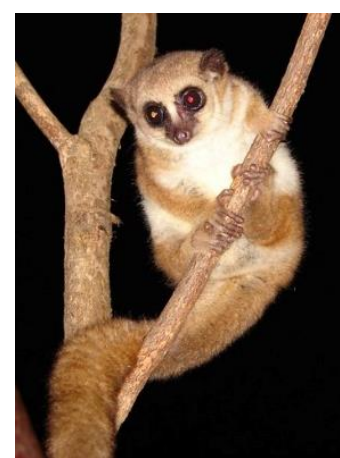

Tsitsiha Cheirogaleus major

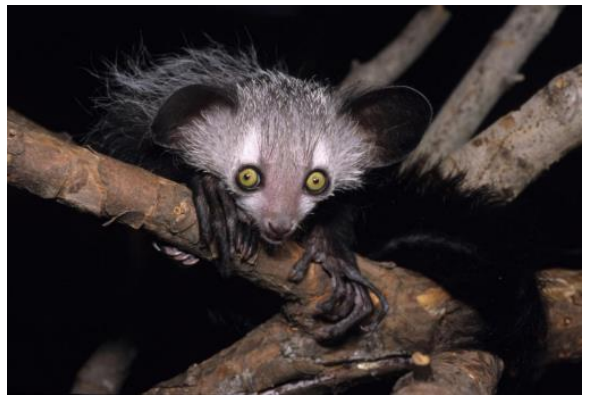

Aye-aye Daubentonia madagascariensis

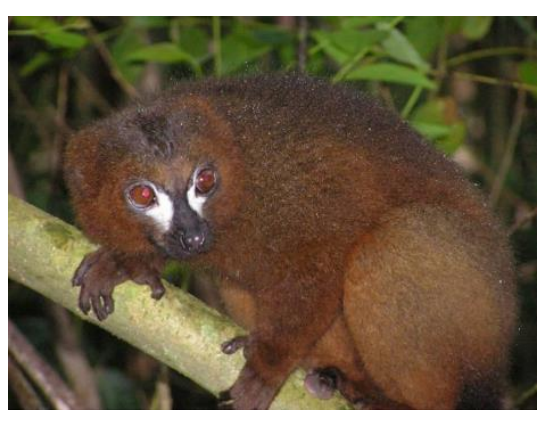

Tongo Eulemur rubriventer 

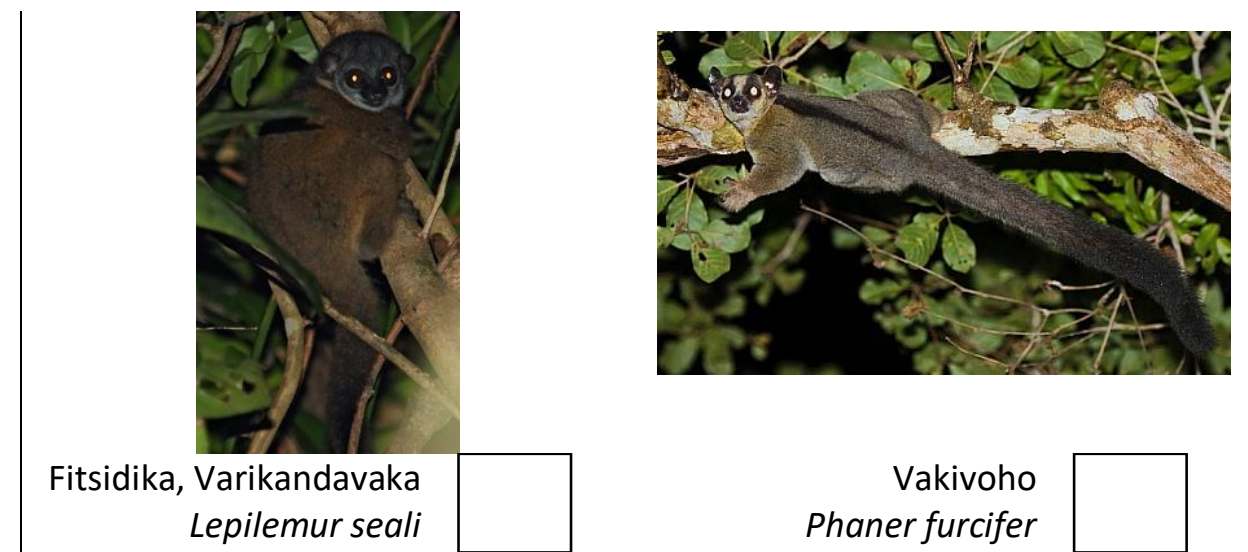

Lepilemur seali

Vakivoho Phaner furcifer 


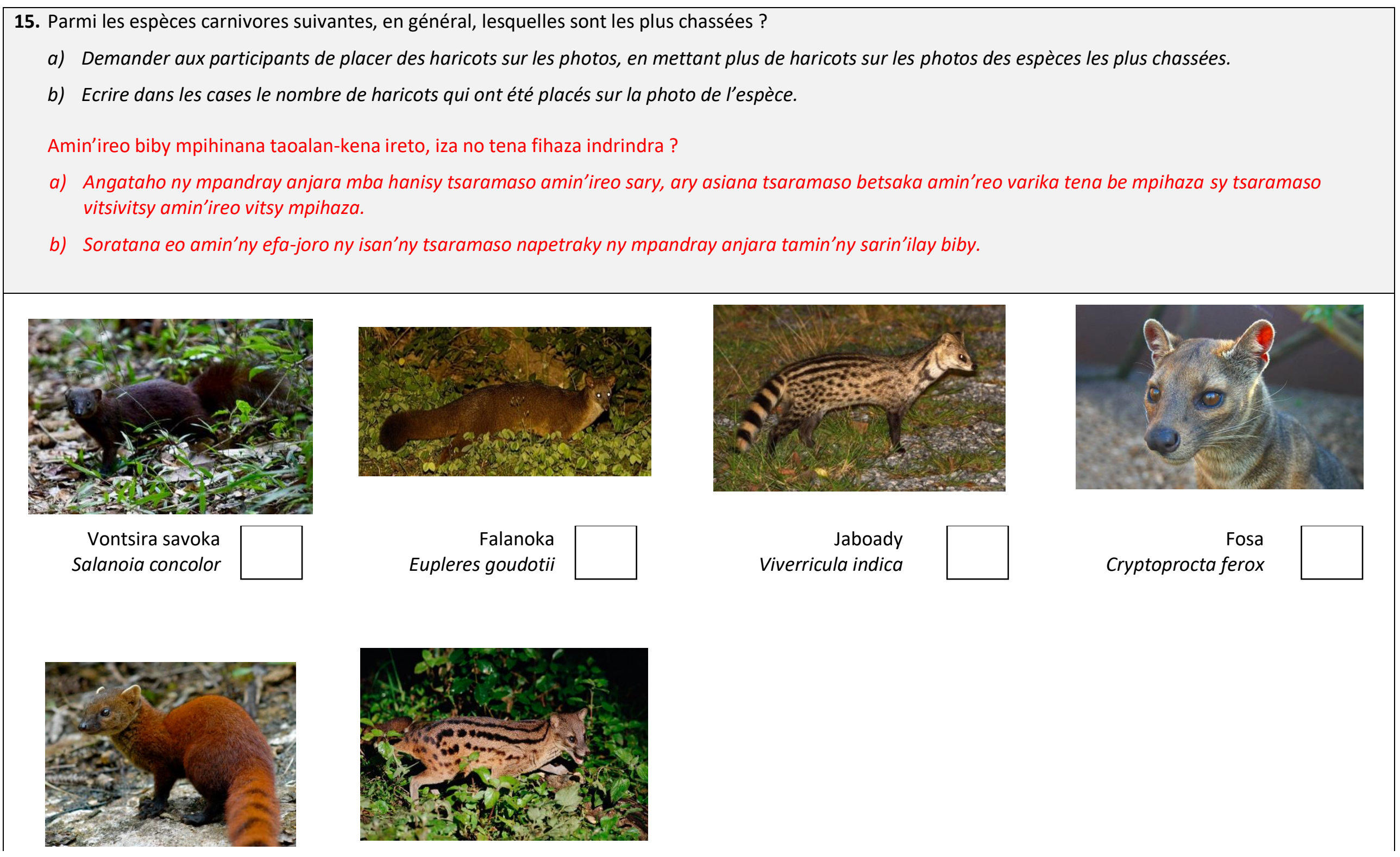


Vontsira

Galidia elegans
Tombokantsodiny

Fossa fossana

16. Parmi les espèces de tenrecs suivantes, en général, lesquelles sont les plus chassées ?

a) Demander aux participants de répartir des haricots sur les photos, en mettant plus de haricots sur les photos des espèces les plus chassées.

b) Ecrire dans les cases le nombre de haricots qui ont été placés sur la photo de l'espèce.

Amin'ireto bibikely mampinono manaraka ireto, iza no tena fihaza indrindra ?

a) Angataho ny mpandray anjara mba hanisy tsaramaso amin'ireo sary, ary asiana tsaramaso betsaka amin'reo varika tena be mpihaza sy tsaramaso vitsivitsy amin'ireo vitsy mpihaza.

b) Soratana eo amin'ny efa-joro ny isan'ny tsaramaso napetraky ny mpandray anjara tamin'ny sarin'ilay biby.

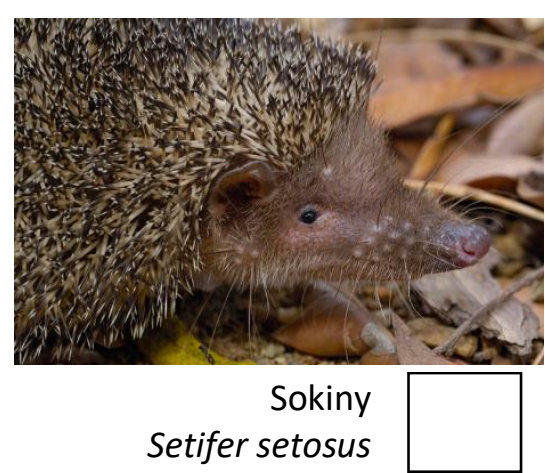

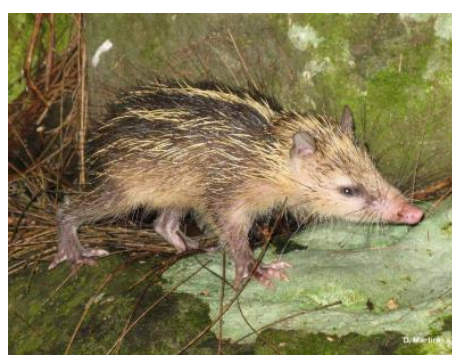

Trandraka Tenrec ecaudatus

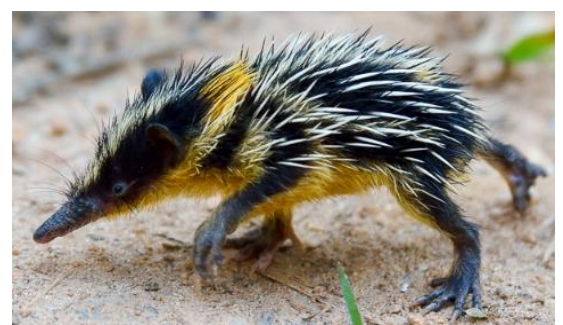

Antsora, Tsora Hemicentetes semispinosus

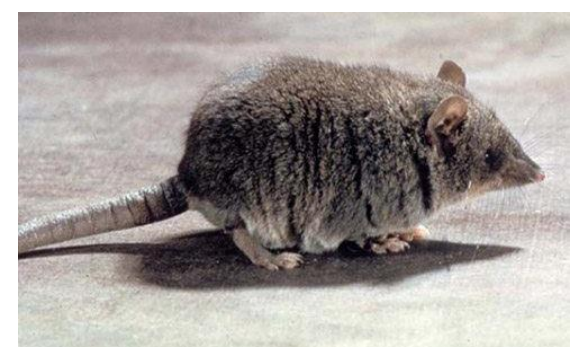

Voalavo anala Microgale sp.

17. Parmi les espèces de chauve-souris suivantes, en général, lesquelles sont les plus chassées ?

a) Demander aux participants de répartir des haricots sur les photos, en mettant plus de haricots sur les photos des espèces les plus chassées. 
b) Ecrire dans les cases le nombre de haricots qui ont été placés sur la photo de l'espèce.

Amin'ireto ramanavy manaraka ireto, iza no tena fihaza indrindra ?

a) Angataho ny mpandray anjara mba hanisy tsaramaso amin'ireo sary, ary asiana tsaramaso betsaka amin'reo varika tena be mpihaza sy tsaramaso vitsivitsy amin'ireo vitsy mpihaza.

b) Soratana eo amin'ny efa-joro ny isan'ny tsaramaso napetraky ny mpandray anjara tamin'ny sarin'ilay biby.

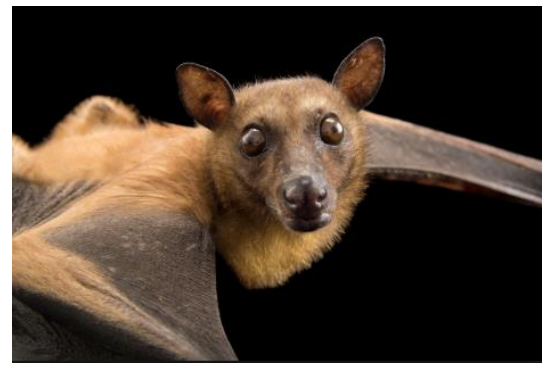

Andrehy

Rousettus

madagascariensis

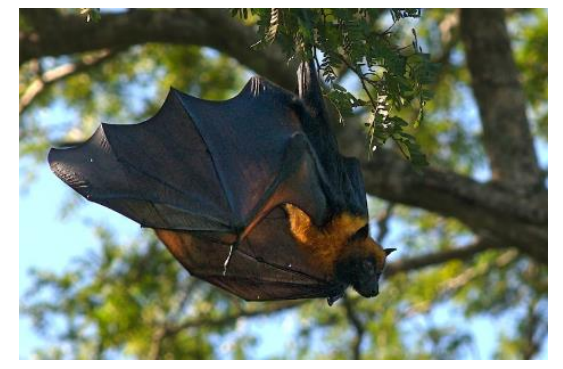

Fanihy

Pteropus rufus

18. A. Identifier les :

- 2 espèces de lémuriens

et

- 1 espèce carnivore :

- 1 espèce de tenrec :

- 1 espèce de chauve-souris :

dont le plus grand nombre de haricots ont été placés sur la photo de l'espèce.

Mariho ireo :

- Varika 2: sy 


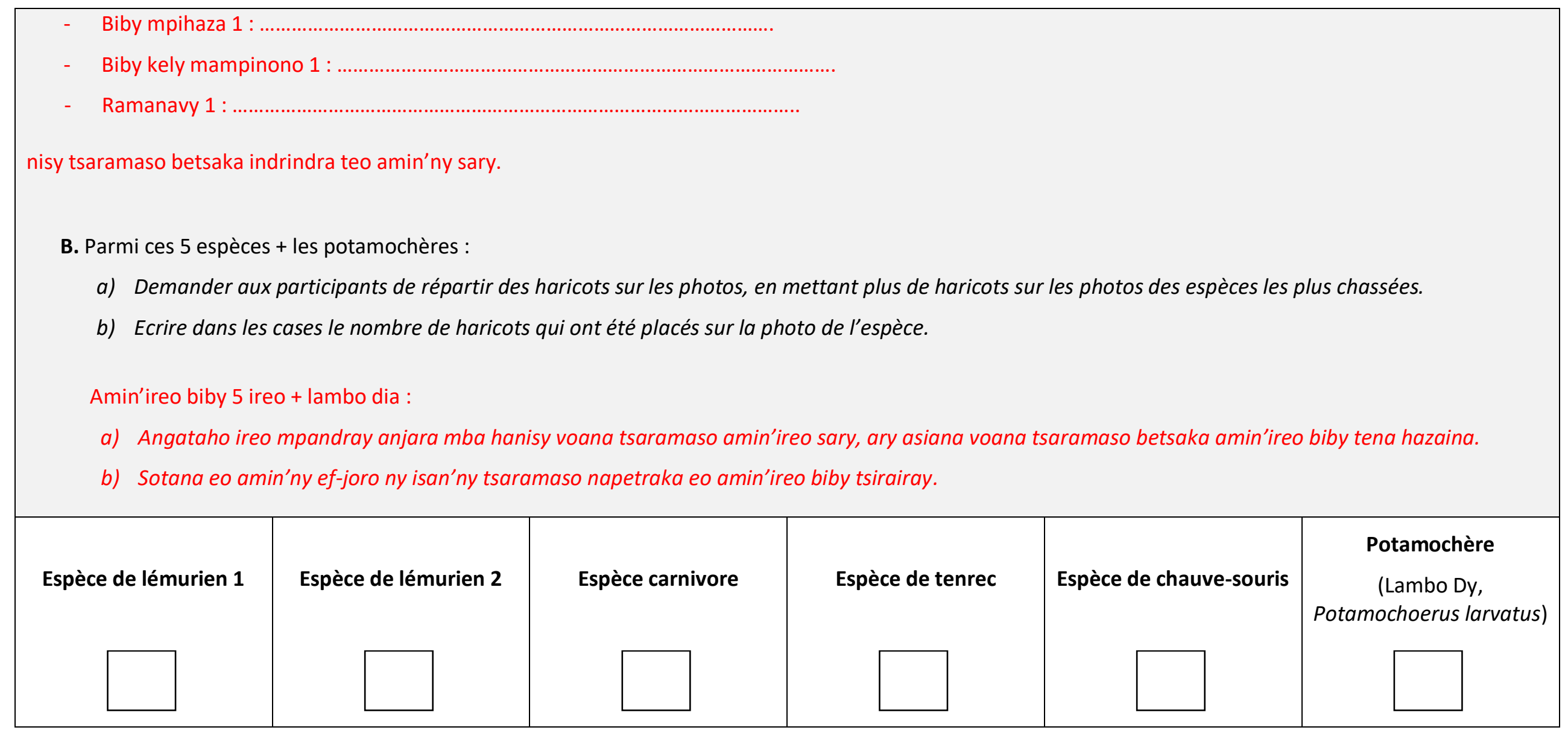




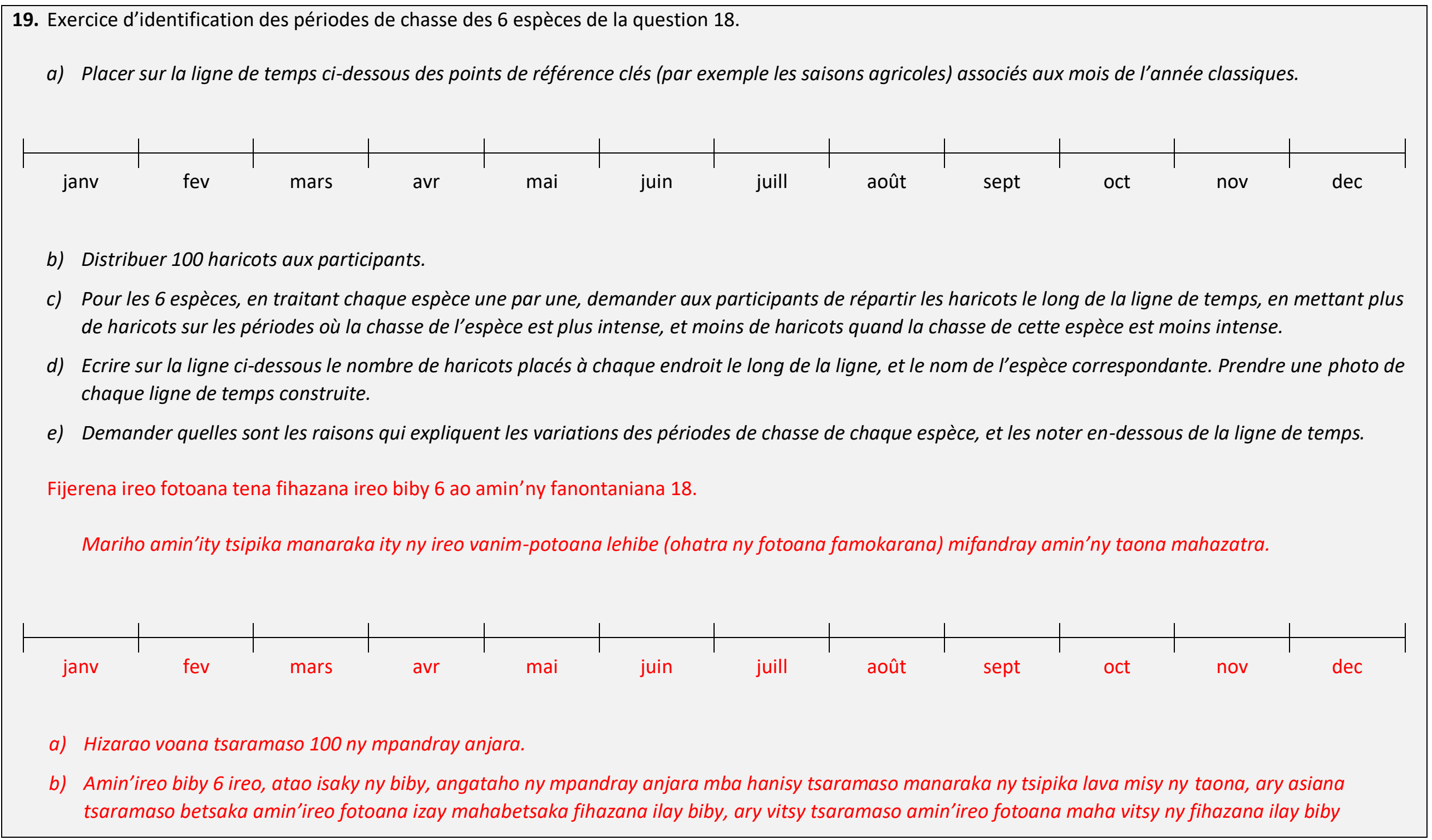


c) Soraty eo ambany ny isan'ny tsaramaso napetraky ny mpandray anjara ami'ireo tsipika ary ny anaran'ny biby mifandray amin'izany. Alaina sary ny tsipika misy ny vanim-potoana rehefa vita izany rehetra izany.

d) Angataho ny mpandray anjara ny antony mahatonga ny fiovana misy amin'ireo fotoana hanaovana fihazana amin'ny biby tsirairay, ary mariho amin'ny tsipika manaraka ity.

\section{Espèce 1}

Biby 1 :

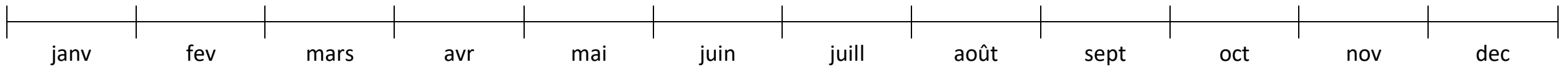

\section{Explications :}

Fanazavana : .

\section{Espèce 2}




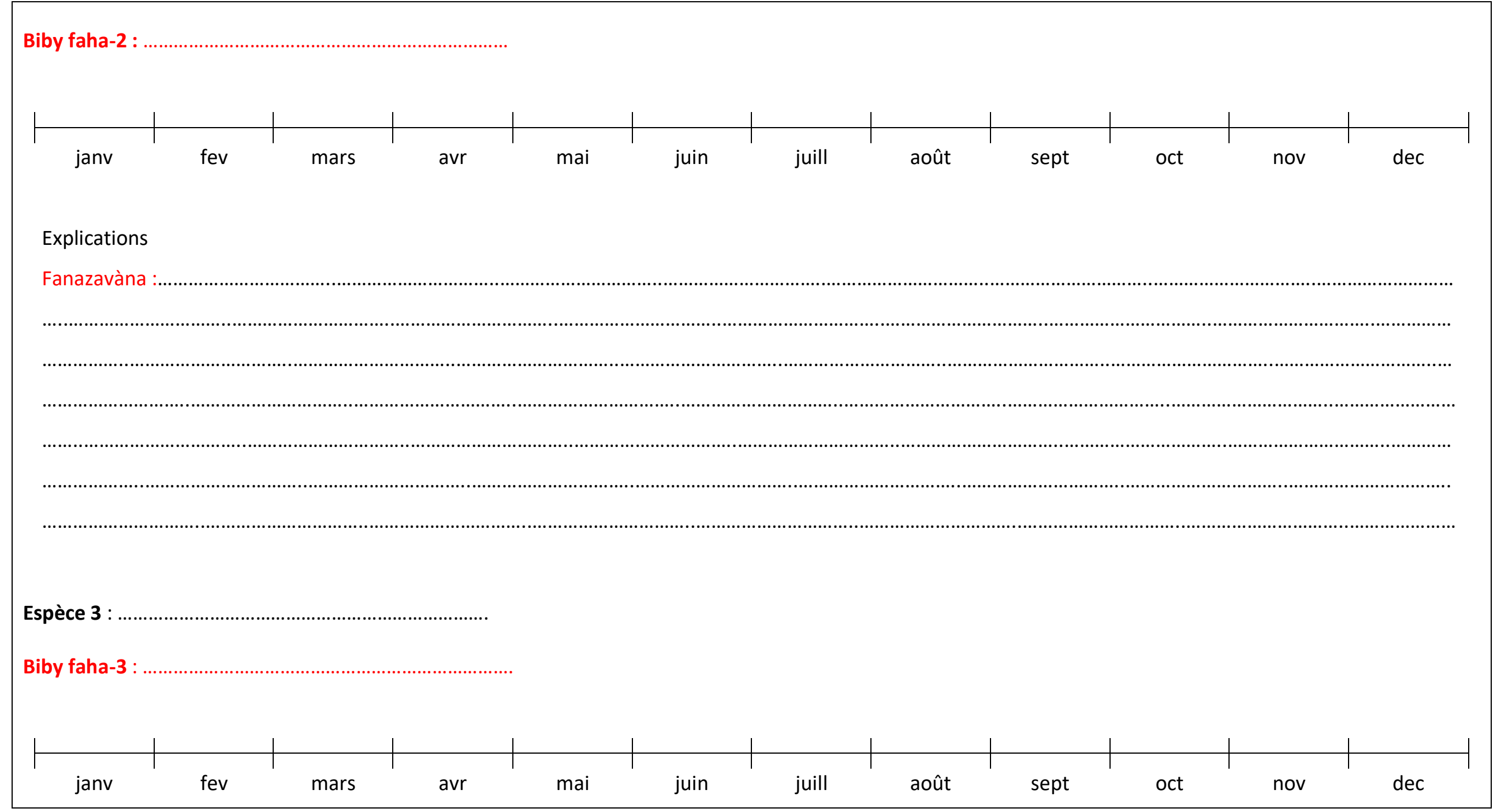




\section{Explications}

Fanazavàna :

(n)

…

(1)

(1)

(1)

\section{Espèce 4}

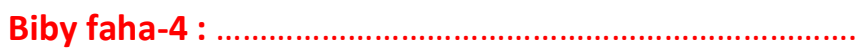

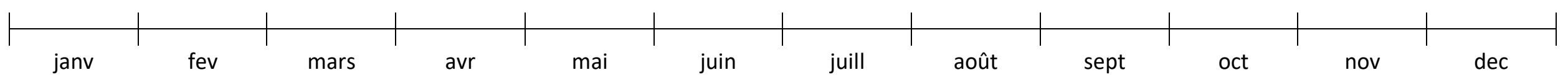

\section{Explications}

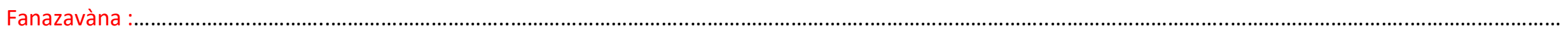
(n) ...........

. 


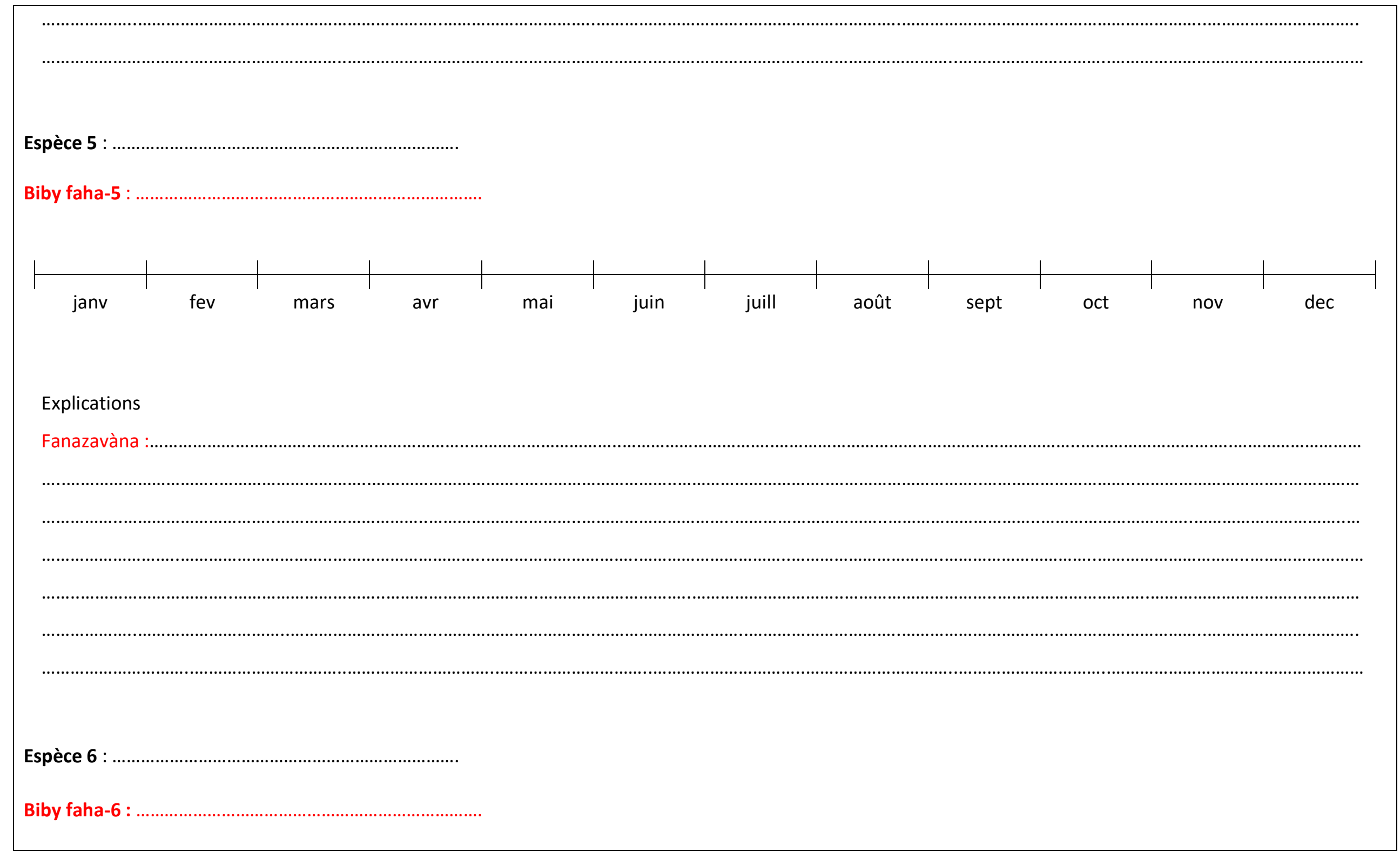




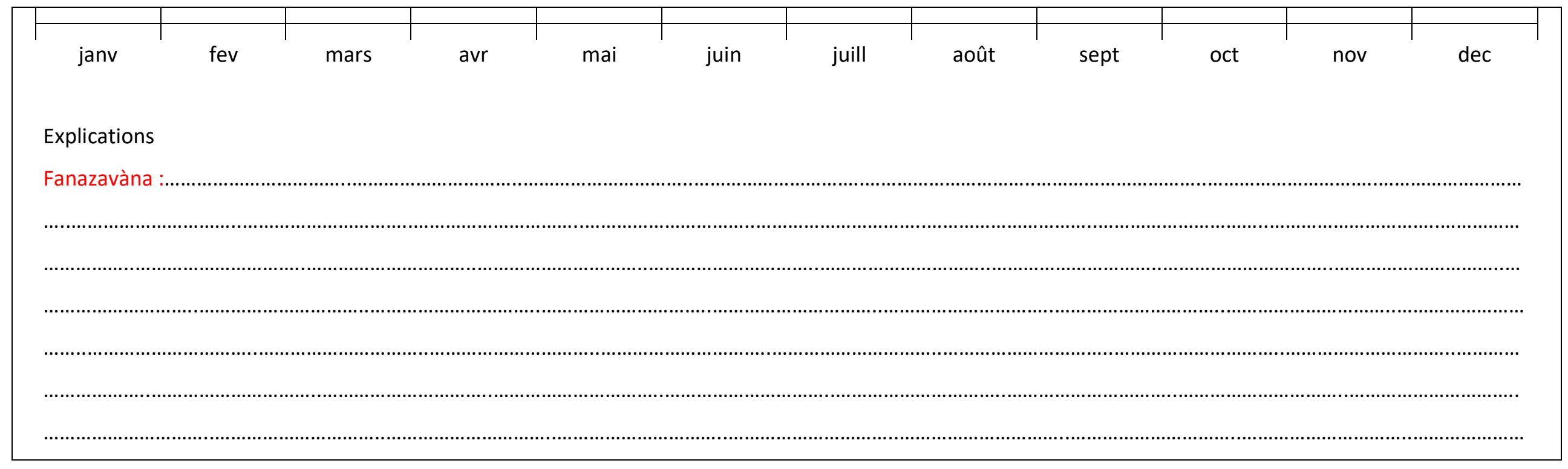




\section{ORGANISATION SOCIALE DES CHASSEURS}

20. Est-ce que vous vous réunissez parfois entre chasseurs du village pour parler de votre activité de chasse ?

Misy fotoana ve ianareo mpihaza eto an-tanàna mivory miaraka mba iresahana mikasina ny fihazàna ataonareo?

21. Si QErreur! Source du renvoi introuvable.0=oui, comment décidez-vous à quel moment vous vous réunissez, et de quels aspects de la chasse parlez-vous pendant ces réunions ?

Raha Q20Erreur ! Source du renvoi introuvable.=eny, ahoana no andraisanareo fanapahan-kevitra ny amin'ny fotoana ivorianareo, sy inona mikasika ny fihazàna no resahanareo mandritra ny fivoriana?

22. Si QErreur ! Source du renvoi introuvable.=non, aimeriez-vous le faire ? Raha QErreur ! Source du renvoi introuvable.0=tsia, tianareo ve ny hanaovana izany ?

23. Si QErreur ! Source du renvoi introuvable.2=non, pourquoi ?

Raha Q22=tsia, satria nahoana?

24. Est-ce que vous vous réunissez parfois entre chasseurs de villages voisins pour parler de votre activité de chasse? lanareo ve matetika manao fivoriana miaraka amin'ireo mpihaza avy amin'ny tanàna hafa mba iresahana momba ny fihazàna?

25. Si QErreur! Source du renvoi introuvable.=oui, comment décidez-vous à quel moment vous vous réunissez, et de quels aspects de la chasse parlez-vous pendant ces réunions?

Raha Q24=eny, ahoana no hanapahanareo hevitra mikasika ny fotoana ivorianareo, ary inona mikasika ny fihazana no tena resahanareo mandritra ny fivoriana? 
26. Si QErreur ! Source du renvoi introuvable.=non, aimeriez-vous le faire ?

Raha tsia, tianareo ve ny hanaovana azy?

27. Si QErreur ! Source du renvoi introuvable.=non, pourquoi ? Raha tsia, satria nahoana? 
ANNEXE 3 - QUESTIONNAIRE DE DISCUSSION DE GROUPE AVEC LES FEMMES DE CHASSEURS SUR LEUR ROLE DANS LES ACTIVITES DE CHASSE AUtOUR DU PARC NATUREL DE MAKIRA

\section{Présentation du groupe de discussion et obtention du consentement des participants}

"Bonjour, nous sommes et Nous faisons partie d'un groupe d'enquêteurs qui effectuent des recherches dans ce secteur pour étudier le rôle des femmes dans les pratiques de chasse.

Votre participation à ce groupe de discussion est volontaire, a été recommandée par les autorités locales qui ont donné leur accord pour procéder à cette étude, et il n'y a ni gain ni perte associée à votre participation ou à votre refus de participer. Cette discussion devrait durer environ 1 heure. Si à un quelconque moment vous n'êtes plus à l'aise de participer, il suffira de nous le signaler et vous pourrez quitter la discussion. Pendant les discussions, n'hésitez pas à nous poser des questions ou à nous signaler si une question n'est pas claire.

Toutes les informations que vous allez donner resteront strictement confidentielles et anonymes, et ne seront utilisées que pour caractériser les pratiques du village dans lequel vous vivez, et serviront de référence pour des études ou des activités futures. Les résultats de cette étude seront utilisés pour évaluer la durabilité des pratiques de chasse dans votre village. Si les pratiques actuelles s'avèrent être non durables, WCS proposera alors aux chasseurs de votre village de les accompagner dans la conception et la mise en cuuve d'activités de conservation qui auront pour but de rendre leurs pratiques de chasse durables, afin que la faune sauvage soit une source durable de nourriture pour la communauté et que les espèces menacées d'extinction soient protégées. Les avantages de l'étude pour les personnes qui participent à l'étude seront donc d'être potentiellement appuyées dans le développement de systèmes de prélèvement durable de la faune dans leur village. Si ces systèmes sont bien appliqués, les générations futures en verront aussi les bénéfices puisqu'elles pourront continuer à reposer sur la chasse pour subsister, sans épuiser les populations d'espèces sauvages. Cela permettra également de maintenir les services écosystémiques assurés par les espèces sauvages pour les générations présentes et futures.

Les résultats de cette étude vous seront présentés dans quelques mois, à une date qui sera convenue avec les autorités de la zone pour garantir la présence des personnes concernées et intéressées.

L'identité des participants aux groupes de discussions ne sera jamais communiquée à quiconque dans ou en dehors du village de quelque façon que ce soit, et nous ne collectons pas votre nom ou prénom pour protéger votre vie privée.

Est-ce que vous comprenez ce que cette enquête implique ? Si oui, accepteriez-vous de participer à cette discussion ? Soyez informé qu'il n'y a pas de bonnes ou de mauvaises réponses, seule votre opinion compte.

Pour plus d'informations vous pouvez contacter Hajaniaina Rasoloarison au 03333181 49. » 


\section{Fanazavana ilay fanadihadiana ary fahazoana ny faneken'ilay olona hohadihadiana}

«Miarahaba anao, ny anarako dia Anisan'ireo vondron'olona mpanao fanadihadiana manao fikarohana amin'ity fizarana ity aho mba hahafantarana ny foto-piveloman'ireo tokantrano, indrindra ireo izay mivelona amin'ny ala voajanahary. Ny fandraisanao anjara amin'izao fanadihadiana izao dia tsy an-tery, ary nahazahoana alàlana tamin'ny manampahefana eto an-toerana mba ahafahana manao izany fanadihadiana izany, ary tsy misy tombontsoa na fatiantoka manokana na handray anjara ianao na mandà ny handray anjara. Mety aharitra X minitra eo ho eo izao fanadihadiana izao izay ataontsika amin'ny toerana mitokana. Raha toa ka tsy mazoto handray anjara amin'ny fahadihadiana ianao dia afaka miala amin'ny adihevitra izay atao. Mandritra ny fanadihadiana, aza misalasala manontany ahy na milaza amiko raha misy fanontaniana tsy mazava aminao. Afaka mandà ihany koa ianao raha misy fanontaniana tsy tianao valiana.

Hanontany fanontaniana vitsivitsy aminao ary aho mikasika ny fomba fihazàna eo anivon'ny tokantranonao. Ny zavatra rehetra holazainao eto dia mijanona ho tsiambaratelo, ary tsy hampiasaina afa-tsy ho famaritana ny fombafomba ao an-tokantrano eto amin'ny vohitra fonenanao, ary amin'ny fikarohana na asa any aoriana any. Ny vokatr'izao fanadihadiana izao dia mba ahafahana mijery maharitra ny fihazàna eto amin'ny faritra misy anareo. Raha ohatra ny fihazana amin'izao fotoana izao dia vinavinaina tsy aharitra, WCS dia hanoso-kevitra anareo mpihaza eto amin'ny vohitra amin'ny fandrosoana soso-kevitra mba ampaharitra izany fihazana izany, mba ahatonga ireo biby fihaza ahazoana sakafo maharitra hoan'ny fokonolona sy mba ahavoaharo ireo biby ahiana ho lany tamingana. Ireo olona izay manaiky hanaovana fanadihadiana izany dia mety ahazo tombony amin'ny fampiroboroboana sy fampianarana ny fihazàna maharitra amin'ny vohitra misy azy. Raha tanterahina tsara ireo rafitra ireo dia handray soa ihany koa ny taranaka ho avy satria afaka manohy miantehitra amin'ny fihazana ireo biby dia tsy lany tamingana. Hanampy koa ny fitazonana ny vokatry ny haivoary homen'ny bibidia ho an'ny taranaka ankehitriny sy ho avy.

Ny vokatr'izao fanadihadiana izao dia hampahafantarina an'ity fiaraha-monina ity afaka volana vitsivitsy. Izany dia atao amin'ny fotoana izay ifanarahana amin'ny manam-pahefana eto amin'ny faritra, amin'ny fotoana hahafahan'izay rehetra liana amin'ny valin'ny fanadihadiana manatrika.

Ny mombamomba ny mpandray anjara amin'ny fanadihadiana iarahana dia tsy holazaina na amin'iza na amin'iza ao amin'ny tanàna na ivelan'ny tanàna hanaovana fanadihadiana na amin'ny fomba ahoana na amin'ny fomba ahoana, ary tsy handray ny anaranareo sy ny mombamomba anareo izahay mba hiarovana ny fiainanareo manokana.

Azonareo ve ilay tanjona tianay ahatongava amin'ilay fanadihadiana ? Raha eny, dia manaiky ve ianao fa hiady hevitra miaraka isika ? Marihana tsara fa tsy misy valimpanontaniana ratsy fa ny fijerinareo sy ny hevitrareo no tena manan-danja.

Raha mila fanazavàna fanampiny dia afaka miantso an'i Hajaniaina Rasoloarison au 03333181 49. » 


\begin{tabular}{|c|c|}
\hline $\begin{array}{l}\text { Date : } \\
\text { Daty : }\end{array}$ & $\begin{array}{l}\text { Nom de la COBA/ZOC: } \\
\text { Anaran'ny COBA/ZOC: }\end{array}$ \\
\hline \multirow{2}{*}{$\begin{array}{l}\text { Prénom et nom du facilitateur de la } \\
\text { discussion : } \\
\text { Fanampin'anarana sy anaran'ny } \\
\text { mpanelanelana: }\end{array}$} & $\begin{array}{l}\text { Village/hameau : } \\
\text { Tanàna/Zana-tanàna : }\end{array}$ \\
\hline & \multirow{2}{*}{$\begin{array}{l}\text { Nombre et sexe des personnes dans le groupe de } \\
\text { discussion (ne pas compter les facilitateurs de la } \\
\text { discussion): } \\
\text { Isan'ny olona sy sokajy ao anatin'ny vondrona } \\
\text { hanaovana fanadihadiana (tsy hisaina ny mpanentana } \\
\text { ny ady hevitra): }\end{array}$} \\
\hline $\begin{array}{l}\text { Prénom et nom de la personne qui remplit le } \\
\text { formulaire : } \\
\text { Anaran'ny olona mpandray an-tsoratra : }\end{array}$ & \\
\hline $\begin{array}{l}\text { Heure de début: } \\
\text { Ora nanombohana: }\end{array}$ & $\begin{array}{l}\text { Heure de fin : } \\
\text { Ora namaranana: }\end{array}$ \\
\hline
\end{tabular}


1. Avez-vous un rôle dans les décisions d'aller à la chasse, de quelle espèce les hommes vont chasser, combien de temps ils vont rester en forêt, là où ils vont chasser, etc. ?

Par exemple, est-ce que vous demandez parfois aux hommes d'aller chasser pour pouvoir avoir de la viande à manger ? Est-ce que vous demandez parfois de capturer une espèce particulière pour pouvoir ensuite la cuisiner ou la vendre?

Expliquer.

Manana andraikitra ve ianareo amin'ny fanapahan-kevitra momba ny fihazàna, ny amin'ny biby hohazain'ny olona, firy andro ny mpihaza no tokony hijanona any anaty ala, ny toerana hanavaovana ny fihazàna, sns. ?

Ohatra, mangataka matetika amin'ireo lehilahy mpihaza ve ianareo mba ahazoana heno hoanina ? Mangataka ihany koa ve ianareo mba hihazàny biby manokana mba andrahoina na amidy ?

Hazavao.

2. Si vous êtes déjà allées chassez avec vos maris, qu'est-ce que vous faites pendant qu'ils chassent ?

(Est-ce qu'elles posent des pièges, vérifient les pièges de sorties de chasse antérieures, collectent d'autres ressources dans la forêt, etc. ?)

Raha efa nihaza niaraka tamin'ny vadinareo ianareo, inona ny asa ataonareo mandritra izany ?

(Manampy mametraka fandrika ve, mitsirika ny fandrika izay napetraka, maka zavatra hafa any anaty ala, sns. ?) 
3. Y a-t-il des espèces particulières que les femmes chassent, ou des techniques ou des outils qu'elles utilisent spécifiquement, ou des moments ou des zones particulières ou elles chassent ? Si oui, les décrire.

Est-ce que ces aspects sont spécifiques aux femmes, ou est-ce que les hommes font pareil ? Expliquer pourquoi.

Misy biby manokana ve hazain'ny vehivavy, na teknika manokana ampiasaina, na fitaovana manokana ihany koa izay ampiasainy, amin'reo toerana sy fotoana hanaovany izany ? Raha eny, hazavao.

Ireo zavatra nohazavaina ireo ve dia indrindra hoan'ny vehivavy, sa mitovy amin'ny fampiasan'ny lehilahy ihany koa ? Satria nahoana. 
4. Quand un ménage veut vendre le gibier capturé, comment est-ce qu'il prend cette décision ? Est-ce plutôt l'homme ou plutôt la femme qui décide de vendre du gibier, ou est-ce que les conjoints prennent la décision ensemble ? Est-ce que cela dépend des espèces ou des quantités que vous allez vendre?

Comme est-ce que vous décidez de la quantité qui va être vendue ?

Est-ce que c'est pareil si l'un de vous veut échanger le gibier contre quelque chose ?

Raha misy fianakaviana iray te hivarotra biby fihaza iray, ahoana no hanapahany hevitra ? Sa indrindra ireo lehilahy sa vehivavy no mandray ny fanapahan-kevitra ny hivarotra ireo biby, sa mandray fanapahan-kevitra miaraka izy mivady ? Miankina amin'ny

Ahoana ny haminavinanareo ny habetsaka'ny hena izay ho amidy?

Mitovy amin'izay ihany koa ve ny fanapahan-kevitrareo raha te hanakalo biby dia ianareo ?

5. Après une vente, comment décidez-vous de la façon dont vous allez dépenser l'argent gagné ? Est-ce plutôt l'homme ou plutôt la femme qui décide, ou est-ce que les conjoints prennent la décision ensemble ? Est-ce que cela dépend du montant d'argent gagné ?

Rehefa vita ny fivarotana, ahoana ny hanapahanareo hevitra mikasika ny handanianareo ny vola hazonareo tamin'ny fihazàna ? Ny lehilahy ve sa ny vehivavy no tena mandray ny fanapahan-kevitra sa iarahana izany rehetra izany ? Sa miankina ihany koa amin'ny habetsaky ny vola azo? 
6. En général, qui dans le ménage cuisine le gibier capturé ? Est-ce que la personne qui cuisine doit avoir l'autorisation de quelqu'un d'autre dans le ménage pour cuisiner ? Est-ce que cela dépend des espèces, de quantités, etc. ?

Amin'ny ankapobeny, iza no mahandro ireo vokatry ny haza ao antrano ? llay olona mahandro azy ve dia tsy maintsy mahazo alalana avy amin'ny olona ao antrano ahandro azy vao manao izany ? Miankina amin'ny biby azo sy ny habeny ve izany rehetra izany, sns. ?

7. Est-ce que la cuisine de la viande de brousse est différente de la cuisine de la viande domestique ? Si vous aviez le choix entre cuisiner de la viande de brousse ou de la viande domestique, laquelle préféreriez-vous cuisinier? 


\section{Expliquer.}

Ny fomba fandrahoana azy ve dia samihafa amin'ny fomba fandrahoana sakafo mahazatra ao antrano isanandro?

Raha asaina miasafidy ianareo amin'ny fomba fandrahoana biby dia sy biby fiompy, iza no tianareo kokoa ny mahandro azy?

Hazavao.

8. Est-ce qu'il y a des espèces ou des morceaux de viande qui sont réservées pour être consommées par les femmes, par les femmes enceintes ou qui allaitent, ou qui sont réservés aux hommes, ou aux enfants?

Misy biby manokana ve na hena izay natokana manokana hoan'ny vehivavy, na vehivavy bevoka na mampinono, na hoan'ny lehilahy irery ihany, na hoan'ny ankizy ? 
9. Est-ce qu'il y a d'autres aspects de la chasse ou de l'utilisation du gibier dans lesquelles les femmes jouent un rôle ? Expliquer

Misy fomba fihazàna hafa ve na fampiasana ny biby dia izay iandreketan'ny vehivavy na andrisan'ny vehivavy anjara bebe kokoa? Hazavao 